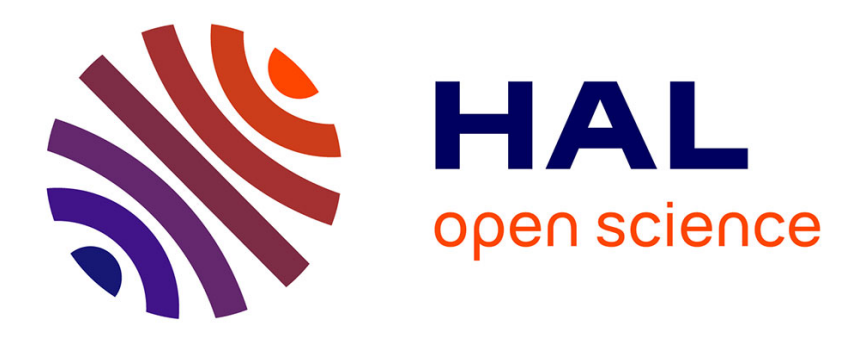

\title{
Characterizing the behavior of sparse algorithms on caches
}

\author{
Olivier Temam, William Jalby
}

\section{To cite this version:}

Olivier Temam, William Jalby. Characterizing the behavior of sparse algorithms on caches. [Research Report] RR-1666, INRIA. 1992. inria-00074891

\section{HAL Id: inria-00074891 \\ https://hal.inria.fr/inria-00074891}

Submitted on 24 May 2006

HAL is a multi-disciplinary open access archive for the deposit and dissemination of scientific research documents, whether they are published or not. The documents may come from teaching and research institutions in France or abroad, or from public or private research centers.
L'archive ouverte pluridisciplinaire HAL, est destinée au dépôt et à la diffusion de documents scientifiques de niveau recherche, publiés ou non, émanant des établissements d'enseignement et de recherche français ou étrangers, des laboratoires publics ou privés. 


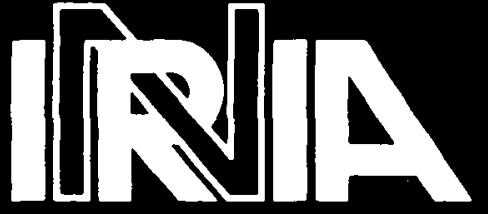

UNITÉ DE RECHERCHE INRIA-RENNES

\section{Rapports de Recherche}

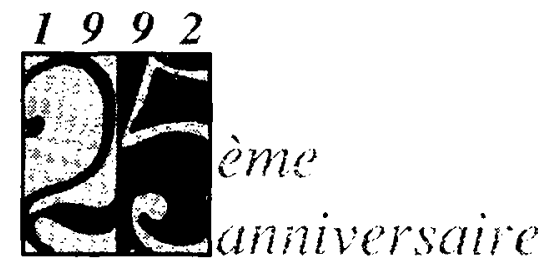

$N^{\circ} 1666$

\section{Programme 1}

Architectures parallèles, Bases de données, Réseaux et Systèmes distribués

\section{CHARACTERIZING THE BEHAVIOR OF SPARSE ALGORITHMS ON CACHES}

Institut National de Recherche en Informatique et en Automatique

Domaine de Voluceau

Rocquencourt B.P.105 78153 Le Chesnay Cedex France

Tél.:(1) 39635511
Olivier TEMAM William JALBY 


\title{
IRISA
}

\section{INSTITUT DE RECHERCHE EN INFORMATIQUE}

\section{ET SYSTEMES ALEATOIRES}

Campus Universitaire de Beaulieu

35042 - RENNES CEDEX FRANCE

Tél. : 99847100 - Télex : UNIRISA $950473 \mathrm{~F}$

Télecopie : 99383832

\section{Characterizing the Behavior of Sparse Algorithms on Caches}

\author{
Olivier Temam, William Jallyy* \\ April 6, 1992 \\ Publication Interne $\mathrm{n}^{\circ} 652$ - Avril 1992 - 20 pages - Programme 1
}

\begin{abstract}
While there are many studies on the locality of dense codes, few deal with the locality of sparse corles. Because of indirect addressing, sparse codes cxhibit irregular patterns of references. In this paper, the bethavior un carthe of onc of the inost frequent primitives SpMxV Simse Matrix-Vector multiply is allalyzed. $A$ model of its refere:uces ix built, and then performance bottlenecks of SpMx are andyacel using model and simulations. Main parameters am.

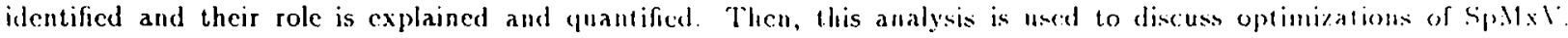
Moreorer a blocking techuigues which takes inlo account the specifies of sparse codes is proprosed.
\end{abstract}

\section{Caractérisation du Comportement des Algorithmes Creux sur les Mćmoires Cacilies}

\begin{abstract}
Résumé Contrairement aux codes denses, peu dectudes traitent de la localité des codes crenx. En raison de: l'adressage indirect, les codes creux exhibent des références irrégulieres. Daus cet article, le comportencut sur le cache

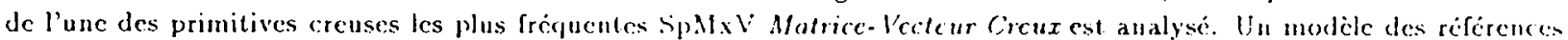

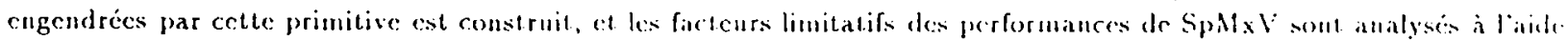
du modèle el de simulations. Les principanx parameleses sont identifiés et leur role est expliqué el quantifie. Puis aetle. analyse est utilisée pour discuter des optimisations de SpMxV. De plus, une methode de blocking prenant en compte les spécificités des codes creux est proposice.
\end{abstract}

Keywords: sparse primitives, caclic, performance prediction, data locality.

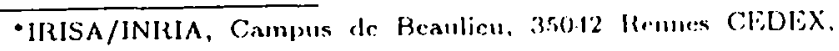

France 
Due to the increasing difference between memory speed and processor speed, it hecomes critical to minimize communications between memory and processor, by addition of caches on the data path. However, a consequence of this worsening difference is that the cost of a cache miss, in terms of processor clock cycles, is becoming quite large, making it critical to improve the hit ratio [17].

Numerical codes are now some of the most demanding progranis in terms of exccution time and memory usage. The existing litterature related to the study of mumerical codes hehavior on carhe memories focuses on regular doloops, i.e with linear references to arrays $[16],[5]$. 'There is an important set of mumerical codes, "sparse codes", which do not belong to this category. Sparse numerical codes like classic numerical codes are made of a collection of simple numerical primitives. We chose to study Sparse Matrix-Vector multiply (SpMxV) because it is among the most frequently used ones along with gaussian elimination [7]. and still it is simple cnough to allow a sharp analysis of ils workings (cf. figure 5); morcover, a number of sparse primitives exhibit rather similar patterns of references (i.e a few arrays addressed regularly and indirect addressing to another array). Because of indirect addressing, sparse codes have the particularity of breeding irregular patterns of references to memory and to cache, and consequently, the behavior of caches under numerical workloads is seemingly non-predictible and hard to analyze. Because of this apparcntly random behavior, caches, which principles rely on locality of programs, are generally said to be incfficient with sparse codes [25].

However, in this paper, we show that this assumption is true culy for a restricted domain of main problem paramet.ers (cache size, line size, matrix bandwidth and number of non-zero elements). Even then, in some cases, it is possible and worthwhile to exploit unused locality through software techniques. 'Though classic blocking methods hardly allow the utilization of this locality (cf. section 5.2.1), it is possible to exploit it through blocking techniques that take into account the specifics of sparse matrices.

In section 2 of this paper, spatial and temporal locality of $S_{p}: M x V$ is qualitatively evaluated and potential problems are identified. Then, in section 3 , a meaningful share of the paper is devoted to modeling the non-regular references which appear in $\mathrm{SpMxV}$, because an unavoidable preliminary to evaluating and optimizing a primitive is a good understanding of its behavior. Besides, the purpose of this section is to show that it is possible to predict the behavior and performance of sparse codes, and to actually quantify their impact on caches. In section 4, using the model and simulations, the behavior of $\mathrm{SpMxV}$ is characterized according to the values of the parameters. Finally, in section 5, software and hardware optimizations are discussed, and a blocking technique based on the observations of the previous section is presented.

\section{A qualitative study of locality within SpMxV}

Position of the problem the purpese of the paterer is the analyze SpMXV on caches. Storagc-by-roul has becen chossent because it is among the most commonly used storage techniques (see page 3 for more details). Otherwise. for salke of simplicity the eache is assumed to be direct-mapped. It can be seen in section 4.4 that this hypothesis is not very. restrictive since set-associative and direct-mapped caches exhibit relatively similar behaviors with $S_{p} M X V$, and l.hal the model built can be extended to sct-rissoctantive cateles (cf. section $\mathrm{A}$ ).

\subsection{Data locality}

A first step to understanding the behavior of $S p M \times V$ on cache is to study the locality of the datid used by this primitive. Since there are $N_{n z}$ references to arrays $X$, Index itmd Matrix, and $2 N$ references to arrays $Y$ and $D$ ), lhe tolial number of references is $3 * N_{n z}+1 * N$ (cf. figure i)

- Arrays $Y$ and $D$ have very similar bohaviors in dir: sense that they both exhibit flawless spatial and timporal locality. Most probably $D(I)$ and $D(I+1)$ will be: stored in registers and therefore should not provoke : reference to memory on each iteration of loop $J$. So arrays $Y$ and $D$ (of size $N$ ) are mostly responsible for $\mathrm{II}$ trinsic misses, and therefore account for a strall slian. of total cache misses (since, in gencral $N \ll N_{n}$ ).

- Arrays Matrix and Index have no temporal locality. and again exhihit flawless spatial locality. These L.wer arrays account for $2 N_{n z}$ references, that is, a major part of total references. Since no element is rensed, cache misses due to these arrays are only zntrinsir misses. Because of their size, they may also prowokr important cross-interfcrences with olher arrays (i.e, flush other arrays from cache).

- Because of the indirect addressing through arriy Index, array $X$ exhibits a complex behavior. If a muiform distribution of non-zero elements on a row withiu the band (of size $W_{B}$ ) is assumed, then the averag. distance between two columns with non-zero elentments is $\frac{W_{B}}{n_{n z}}$. Therefore, in most cases lihere is some sprat tial locality if $\frac{W_{B}}{n_{n}}$ is of the order of L.s. Actually. in usual finite-elcment matrices, the non-zero elements.s are sometimes grouped along specific diagonals, within the band (cf. [8] and figure 6 ). In that case, the spalial locality may not be negligeable even if $\frac{W_{B}}{n_{n z}} \gg L_{s}$

Array $X$ is the only array which presents an uncxploited temporal locality, and therefore from which significant gains can be expected; however the tomporial locality of $X$ is non-trivial and therefore hard to alldlyze and exploit. That is why our cfforts will mainly focus on analyzing the behavior of array $X$. Due to the properties of sparse matrices (especially finte-ele $m e m$ I ones), if there is approximately $n_{n z}$ non-zero clements per row, there is also about $n_{n z}$ non-zero elements per column. A first consequence of that olservation is that 


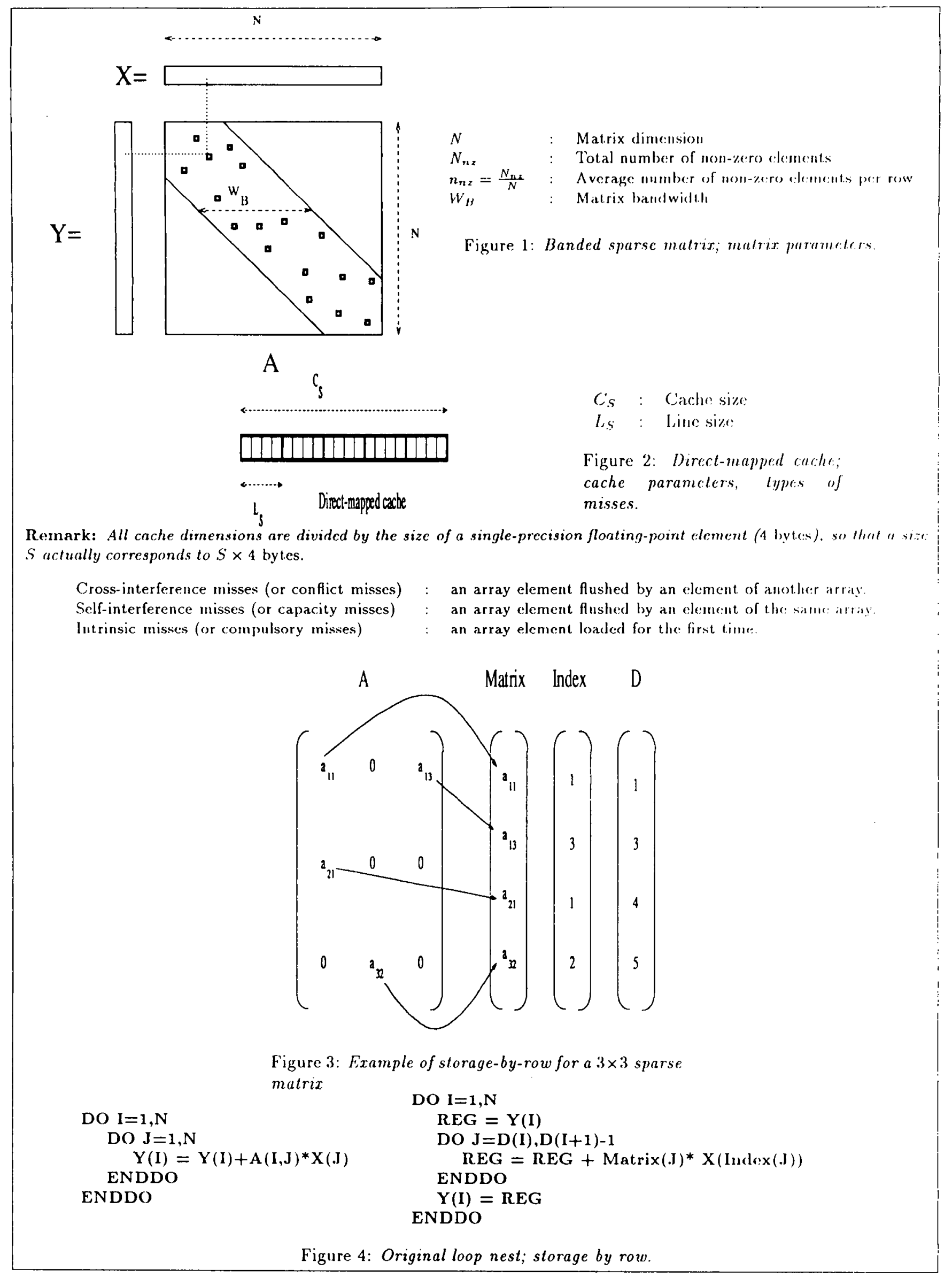

Figure 5: Problem parameters 


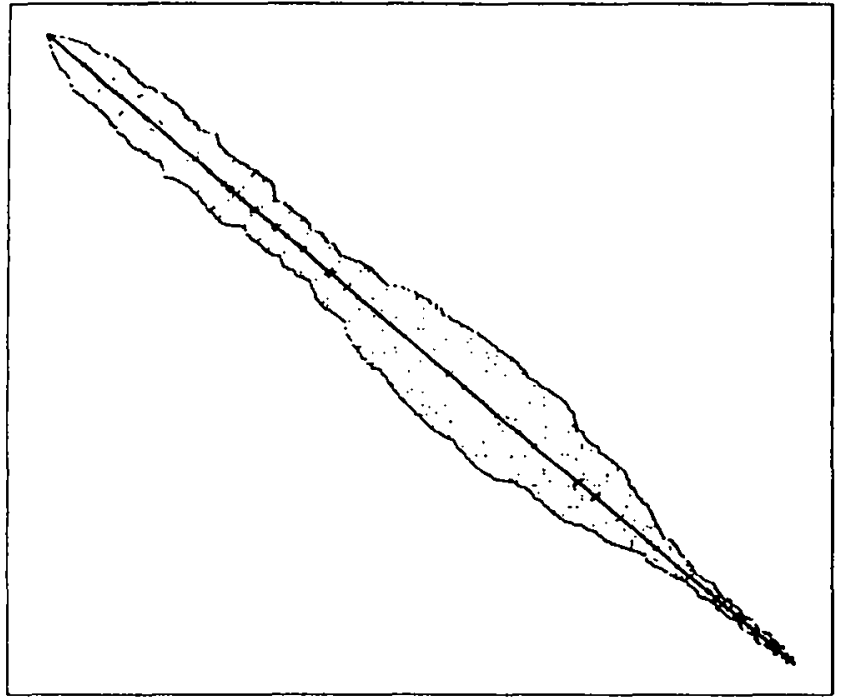

Higure 6: Example of distribution of non-zero elements within finite-clement inatrices (matrix 1138 BUS of the Harwell-Boeing suile).

each elcment of $X$ may theoretically be reused $n_{n z}$ times at best. Secondly, the average distance (in terms of iterations of loop $I$ ) between two reuses is approximately $\frac{W_{B}}{n_{n s}}$. Meanwhile, about $\frac{W_{B}}{n_{n z}} \times 3 n_{n z}$ elements (from arrays $X$, Matrix and Index) are loaded into caclic and may flush the elements to be reused.

The conclusion of the previous observations on $X$ is that whether temporal locality and spatial locality of $X$ are significant and can be exploited highly depends on $W_{B}, C_{S}, L_{S}$ and $n_{n z}$.

\section{Modeling: understanding and quantifying}

From previous section, it appears that the main source of cache misses are misses of Malrix and Index which can be evaluated easily becausc they are intrinsic misses, and misses of $X$ which are hard to estimate because addressing to array $X$ is indirect and irregular. The misses of $X$ are mainly cross-interference or self-interference misses. First of all, the simulations done (cf. section 4) show that the role of cross-interference and self-interference phenomenons on $X$ is very similar. Second, both of the two kinds of misses can be modeled using techniques presented thereafter. Third, the purpose of our model is to provide a good understanding of the interactions between parameters rather than an accurate formula of the total number of cache misses. Therefore, for sake of simplicity, only self-interference misses are precisely modeled and quantilied (cf section 3.1), while only a gross estimate is given for cross-interference misses ( $c$ f. section 3.2 .2 ).

\subsection{Modeling self-interferences of array $X$}

References to array $X$ are highly irregular and consequently carnot be investigated through classic deterministic methods. Therefore, probabilistic modeling is being used. The main problem secms to choose a distribution which matches that of non-zero clements on a row within the band. Thongh it is for the least possible to find an approximate distribution for finite-element matrices, this yields formulas which are too complex to handle (cf. section $A$ ). 'Therefore, though most computations are conducted for any distrihution $p(i, j)$ (probability that clement $(i, j)$ of $A$ is non-zero), uniform distribution $(p(i, j)=p)$ is employed for final calculi. The object of section 3.1 is t.o build the model of references to array $X$ and compute the number of cache misses. 'This part needs not be read thoromghly by anyom: not interested hy model claboration, though it provides an insight on the behavior of $S_{p} M \times V$

\subsubsection{Reducing problem $P$ to problem $\rho^{\prime}$}

Let us consider original matrix $A$. All mon-zero elements of $A$ located on column $j$ of this matrix breed a reference to element $j$ of $X$. Now, let us consider the $e^{\text {th }}$ cache location All elements $j$ of $X$ such that $j \bmod C_{s} \in\left[c, c+l_{s}-1\right]$ an mapped to the same cache line c. 'Therefore, all columuss $j$ of $A$ such that $j \bmod C_{S} \in[c, c+L, s-1]$ breed references to elements of $X$ which are mapped to the same cache lium.

Therefore, it is possible to divide the problem into $\frac{C s}{1 . s}$ set.s of elements of $X$, all elements within a set being mapped in the same cache line. Similarly, $A$ is divided into sets of columns, all breeding references to elements of $X$ ullapind to the same cache line (cf. figure 7). Since, llue cuclus is direct-mapped, none of these sets interact with each other

So, if the original problem can be formalized as followin

Problem P: Compute an approximation of $N_{\mathrm{r} m}^{\mathrm{s} i \mathrm{l}}=$ $N_{c m}(P)$, the number of self-interference misses of array $X$ in the sparse matrix-vector multiplication. The dimension of $X$ is equal to $N$. $A$ is an $N \times N$ matrix, and line cach. is direct-mapped and of size $C_{S}$

Then $P$, is now equivalent to $C_{S}$ subproblems $P_{i},\left(1 \leq i \leq \frac{C_{s}}{L_{s}}\right)$. Through simulations, it is possible to check that, for distributions occuring in finite-clemrnl matrices (non-zero elements are grouped along three diagonals) and even more for uniform distributions, it is a very fair approximation to assume that all subproblems $P_{i}$ are equivalent.

Problem $P^{\prime}$ : Compute an approximation of $N_{\mathrm{cm}}\left(F^{\prime}\right)$, the number of self-interference misses of array $X^{\prime}$. The dimension of $X^{\prime}$ is equal to $N^{\prime}$ (where $N^{\prime}=\left\lfloor\frac{N^{\prime}}{C_{S}}\right\rfloor \times L_{S}$ or $\left.N^{\prime}=\left(\left\lfloor\frac{N}{C_{s}}\right\rfloor+1\right) \times L_{S}\right)$. A is an $N \times N^{\prime}$ matrit; and the cache is direct-mapped and of size. I.S

Then, the number of cache misses of $P$ is approximatioly equal to the number of cache misses of $l^{\prime}$ timus $C_{s}$ :

$$
N_{c m}^{\prime}(P) \simeq \frac{C_{S}}{L_{S}} \times N_{c m}\left(P^{\prime}\right)
$$

\subsubsection{Reducing problem $P^{\prime}$ to problem $P^{\prime \prime}$}

As it has been specified above, problem $P^{\prime}$ is composed of a cache of size $L_{S}$, an array $X^{\prime}$, and a. $N \times N^{\prime}$ matrix $A^{\prime}$ 


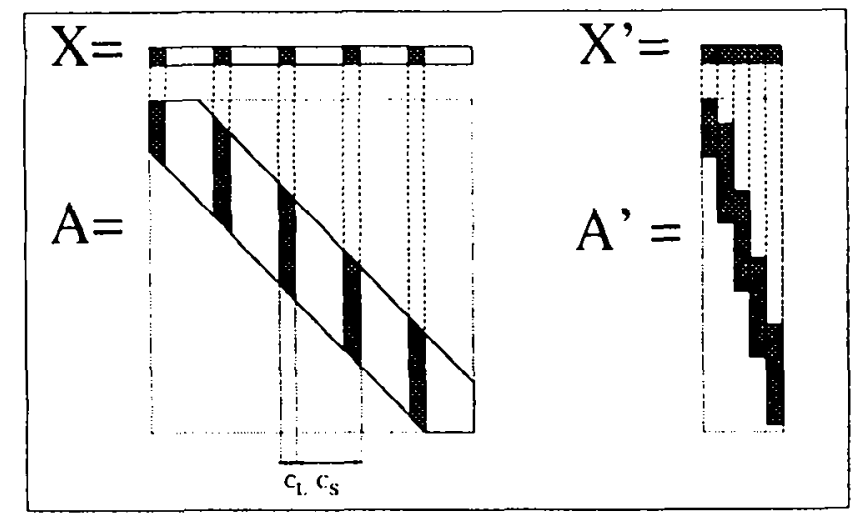

Figure 7: Original problem $P$, and subproblem $P^{\prime}$

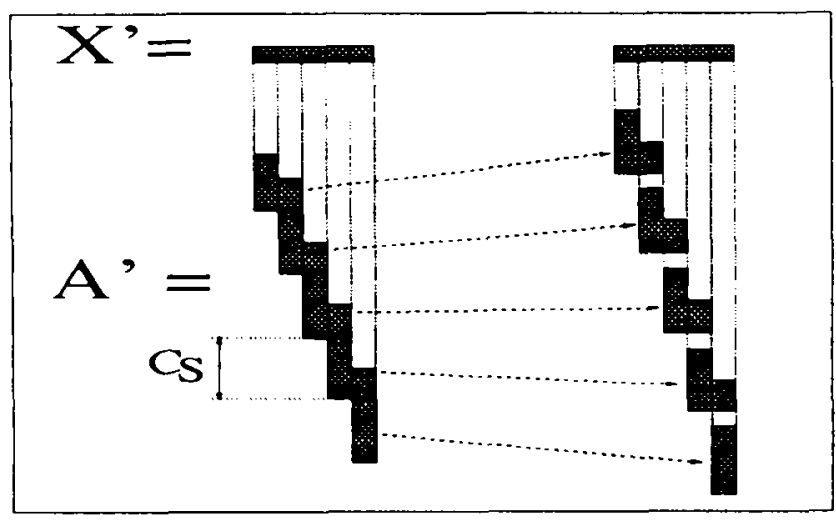

Figure 8: Decomposition of subproblem $P^{\prime}$

Now, $A^{\prime}$ can be decomposed into sections of $C_{S}$ rows, which would all have the same shape as shown on figure 8 . Since, in general, $C_{S}<N$, there is a great number of such sections in $A^{\prime}$ (approximately $\frac{N}{C_{s}}$ ). Through experiments, it can be olsesved that the number of cache misses corresponding to the execution of each section becomes rapidly stable. Therefore, it is possible to restrict the study to only one section $S^{\prime}$ of $A^{\prime}$. Then, the number of cache misses due to $A$ is approximately equal to that of one section times $\frac{N}{C_{S}}$.

Let is do an ultimate simplification. The number of columns in the sections described above is not constant, because of the banded shape of the matrix. In order to ease the computations even more, a section $S^{\prime}$ can be divided into two parts $A_{1}^{\prime \prime}$ and $A_{2}^{\prime \prime}$, each with a constant number of columns (cf. figure 9). The characteristics of each subsection are the following ones

$$
\begin{aligned}
& A_{1}^{\prime \prime}\left\{\begin{array}{l}
n_{c}^{1}=\left\lfloor\frac{W_{B}}{C_{S}}\right\rfloor \\
n_{l}^{1}=C_{S}-W_{B} \bmod C_{S}
\end{array}\right. \\
& A_{2}^{\prime \prime}\left\{\begin{array}{l}
n_{c}^{2}=\left\lfloor\frac{W_{B}}{C_{S}}\right\rfloor+1 \\
n_{l}^{2}=W_{B} \bmod C_{S}
\end{array}\right.
\end{aligned}
$$

Problem $P^{\prime \prime}$ can now be defined as follows:

Problem $P^{\prime \prime}$ : Compute an approximation of $N_{c m}\left(P^{\prime \prime}\right)$, the number of self-interference misses of array $X^{\prime \prime}$. The dimension of $X^{\prime \prime}$ is equal to $N^{\prime \prime}$ (where $N^{\prime \prime}=n_{c}$ ). $A^{\prime \prime}$ is an $n_{l} \times n_{r}$. matrix, and the cache is direct-mapped and of s2it: $L_{S}$.

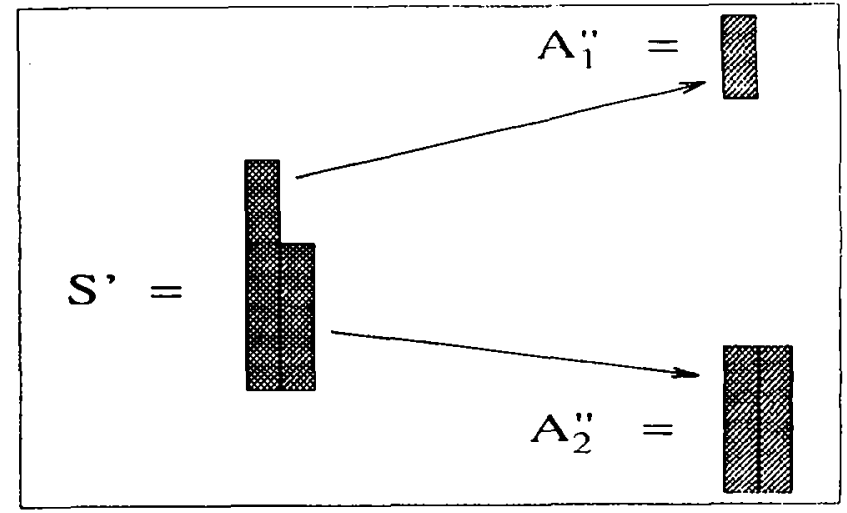

Figure 9: Decomposilion of subproblem. P'

\subsubsection{Estimating the number of cache: misses}

Let us now formalize the notion of "cache miss" within the

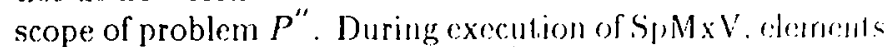
of a subsection $A^{\prime \prime}$ of problem $P^{\prime \prime}$ are referenced row-wior. Let us call $\pi_{\text {out }}^{k}(i, j)$ the probability that ctement $k$ of $\lambda^{\prime \prime}$ be out of cache right before element $(i, j)$ of the subserelinn is referenced. Let us also call $\nu(i, j)$ the probibility thil element $(i, j)$ of $A^{\prime \prime}$ be a non-zero eloment. Now, the probability that element $j$ of $X^{\prime \prime}$ is not in cache, right bef(sere t. ement $(i, j)$ of $A^{\prime \prime}$ is being considered, is equal to $\pi_{m u}^{j}(i, j)$. 'Therefore, the probability for a cache miss lo roceur al thal. moment is equal to $p(i, j) \times \pi_{o u t}^{j}(i, j)$.

Then, the number of cache nuisses due $10, A^{\prime \prime}$ is givell liy. the following expression:

$$
N_{c m}\left(P^{\prime \prime}\right) \simeq \sum_{i=1}^{n_{l}} \sum_{j=1}^{n_{c}} \pi_{o u t}^{j}(i, j) \times \mu(i, j)
$$

And, the total number of cache misses is eynal loo (cl. ser tion $\Lambda$ for more details):

$$
\begin{aligned}
N_{c m}(P) & \simeq \frac{C_{s}}{L_{s}} N_{c m}\left(P^{\prime}\right) \\
& \simeq \frac{C_{s}}{L_{s}} \times \frac{N}{C s} N_{c m}\left(P_{1}^{\prime \prime} \cup P_{2}^{\prime \prime}\right) \\
& \simeq \frac{N^{\prime}}{L_{s}} \times\left(N_{c m}^{\prime \prime}\left(P_{1}^{\prime \prime}\right)+N_{c m}^{\prime}\left(P_{2}^{\prime \prime}\right)\right)
\end{aligned}
$$

An explicit and simple expression of $N_{c m}(P)$ can be dorived easily only if $p(i, j)$ is constiant, i.c if distribution is uniform. Nevertheless, since the goal is to got a hint at the interactions between parameters rather than a precise approximation of the number of cache Inisses, it. is a garol tradeoff to assume that distribution is unform (cf. sertion 3.3 for more details).

So, if distribution is uniform, then $p(i, j)=l$ and $p$ call be given as a function of problem parameters $p=1-(1-$ $\left.\frac{N_{a c}}{N W_{B}}\right)^{L s}$ (cf. section 3.3 for more details). For this distribution of probability, the total number of self-interference misses on $X$ is given by expression of figure 10 .

\subsection{Number of cache misses for each type of misses}

\subsubsection{Self-interferences of array $\lambda$}

$\frac{W_{B}}{C S}>1$ : Since $N_{n z} \ll N \times W_{B}$ when $W_{B}$ is sufficiently. large, it can be assumed that $p \ll 1$ and that $p \simeq$ 


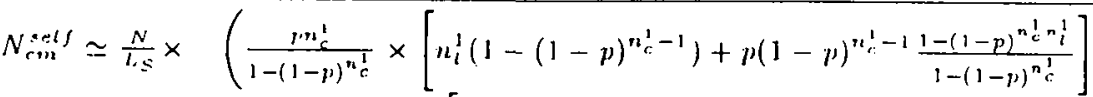

$$
\begin{aligned}
& \left.+\frac{p n_{c}^{2}}{1-(1-p)^{n_{c}^{2}}} \times\left[n_{l}^{2}\left(1-(1-p)^{n_{c}^{2}-1}\right)+p(1-p)^{n_{c}^{2}-1} \frac{1-(1-p)^{n_{c}^{2} n_{l}^{2}}}{1-(1-p)^{n_{c}^{2}}}\right]\right)
\end{aligned}
$$

Figure 10: Expression of the Lolal number of self-interference misses on array $\lambda$

$\frac{N_{n}}{N W_{B}} \times L_{S}$. Therefore, a first order developrnent of expression in figure 10 gives (cf. section $A$ for more detiails)

$$
N_{c m}^{s c l f} \simeq N_{n:}-\frac{N_{n 2}^{2} \times C_{S}}{2 \times N \times L_{S} \times W_{B}}
$$

$\frac{W_{B}}{C=}<1$ : In that case, there are no self-interferences of array $X$ because all active elements of $X$ fit in the caclse, therefore

$$
N_{c m}^{s c l s}=0
$$

\subsubsection{Cross-interferences with array $X$}

A second class of misses breeded by array $X$ are the crossinterference misses between arrays Malrix, Index and arriay $\lambda$.

let us first consider the case where $W_{B}>C_{S}$ (which is the assumption made up to now). Because of their flawless spatial locality, the elements of Matrix and Index can be seen as two trains of references being translated along the cache. Each time one of these sets of references reaches a cache location, it flushes it. Therefore, considering that there are $\frac{N_{n}}{N}$ non-zero clements per row, $2 \frac{N_{n s}}{N}$ elements of Matrix and Index are loaded for each iteration of $I$, and consequently, each cache location is flushed $\frac{2 N_{\text {na }}}{C S N}$ times per iteration of $l$

Therefore, the probability that Matrix or Index flush a given cache line for one iteration of $I$ is peross $=\frac{2 N_{m,} \times L_{s}}{C_{s}}$.

Consequently, since there are $N_{n z}$ references to array $X$, the number of cross-interference misses on $X$ account for

$$
N_{c}^{\text {cross }} m=p_{\text {cross }} \times N_{n z}=\frac{2 N_{n z}^{2} \times L_{S}}{C_{S}}
$$

1t must be noted that, when $L_{S}$ is sufficiently large, the efrect of self and cross-interference misses do not cumulate but become redundant.

The effect of cross-interference misses on other arrays is generally negligeable.

\subsubsection{Intrinsic misses}

There are $N$ references to $D, Y$ and $X$, and $N_{n z}$ references to Matrix and Index. Therefore, the total number of intrinsic misses is given by

$$
N_{c m}^{i n t}=\frac{3 N+2 N_{n z}}{L_{S}}
$$

Since in general $N_{n z} \gg N$, arrays Matrix and Index represent the main source of intrinsic misses.

\subsection{Model accuracy and validity}

In order to understand the soundness and precision of the model, let us first analyze the approximations inade, and then describe some experimental results obtaincel througl simulation.

\subsubsection{Discussing approximations}

During the elaboration of the model, a mumber of approxi mations have been performed:

- The width of the band is considered to br: enjual ter, $W_{B}$ on any row of the matrix, which is met true: for $i \leq \frac{W_{B}}{2}$ and $i \geq N-\frac{W_{B}}{2}$. However, a good tradeoff $f_{0}$ overcoming this problem is to consider that, the artinal number of rows is $N-\frac{w_{u}}{2}$ instead of $N$

- The number of non-zero clements on a row is assumand to be constant. This is not an important approximliation since this fact is relatively true for a great. range of applications, especially for finte-clement matrices

- The number of cache misses is considered to be the: same in all sections $S^{\prime}$ of $A^{\prime}$. This approximation does not affect much the model cither. All experiments done proved the good regularity of the mumber of cache misses across the different sections. 'The larger the matrix, the better is the approximation, because this number becomes stable after a frew sections only. Most problems have very large dimemsions which misliv this start-up effect negligeable.

- The main approximation probably lays in the distribution. Clearly, it appeared that uniform distributuon does not always fit very precisely. This is especially true when cache line $L_{S}$ is equal to 2 (16 bytes), because of the particular spatial locality of finite-element matrices (cf. figure 16). However, for most cases it provides a very good hint at the influence of each parameter. Besides, it is not too easy to understand the distributions of non-zero elements in real sparse matrices, and little litterature could be found on thit1. subject. 'Therefore, approximate distributions had to be designed for some matrices, such as finte-clemenl ones, and hopefully, these distributions proved to give a good description of real finite-clcment matrices. Ilowever these more complex distributions do not allow t.lu computation of an analytical expression of the number of self-interference misses ( $p(i, j)$ is not constant). Parl of the formula obtained would have to be computed ummerically (cf. section $A)$. 


\subsubsection{Experimental testing of the model}

In order to check the precison of the estimate, a cache simulatior has been used.

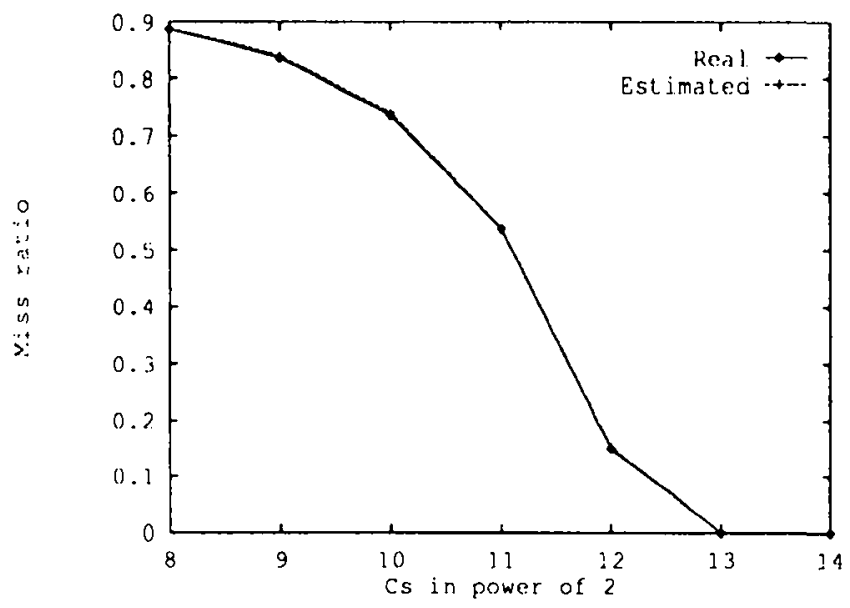

Figure 11: Varying $C_{S}$ : estimated and real number of cache misses

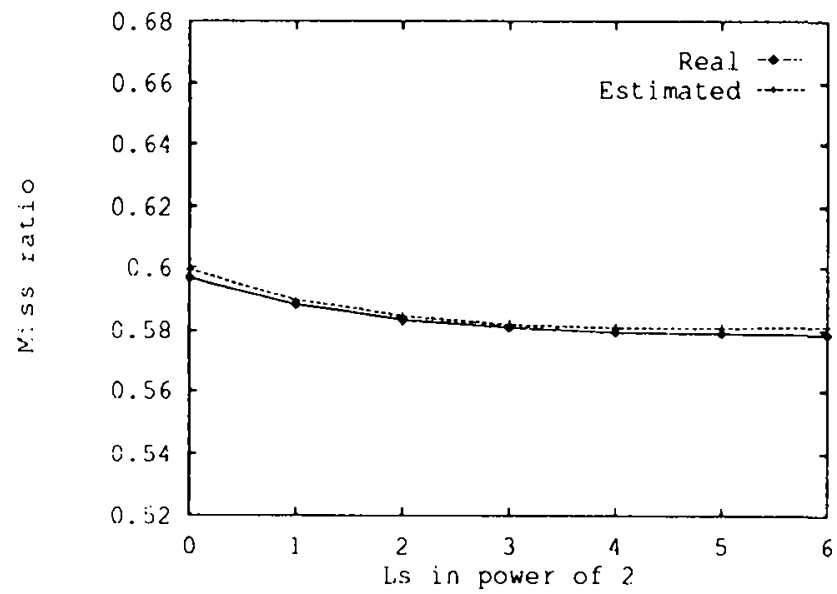

Figure 12: Varying $L_{S}$ : estimated and real number of cache masses

Randoinly generated matrices In addition to this simulator, a subroutine for generating sparse matrices with various distributions has been written. All parameters of cache and matrix can be varied in any domain. In order to get meaningful results, the number of cache misses presented is a mean value obtained through several experiments $(\simeq 100)$ on different sparse matrices with identical characteristics (cf. figures 11, 12).

The parameters of the matrices used for the two graphs of this section are the following ones

\begin{tabular}{|l|l|l|l|l|l|}
\hline$N:$ & $n_{n z}$ & $W_{B}$ & $C_{S}$ & $L_{S}$ & $D_{A}$ \\
\hline $10^{5}$ & $10^{6}$ & 5001 & 2048 & 2 & $1($ or $L R U)$ \\
\hline
\end{tabular}

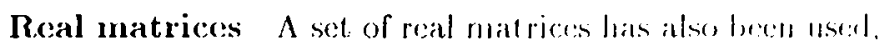

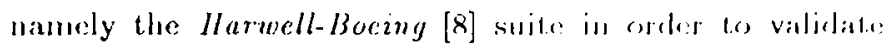
the applicability of our model. It is this set of experiments which showed it was necessary te take inte aceomul more complex distributions than uniform oness esprecially for small line sizes (cf. figure 16). Though some applications still present matrices with close to uniform distributions, especially after using remumbering techuicuess surht as minimum degrec algorithm (cf. figure 22)

\section{Highlighting the role of the problem parameters}

Of course, all problem parancters lave an impact on Sir $\mathrm{MxV}$. However, through moded analysis and experiments. three coefficients $\left(L_{S}, w=\frac{W_{u}}{C_{c}}, d=\frac{n_{u}}{W_{u}}\right)$ proved to have a major impact on the hit ratio, and are sulficicut to chatsacterize most phenomenons. $L_{, s}$ is a critical farandeter, mainly because of its influence on inlrinsic misses, but alsr, on cross-interference misses. Parameder $w=\frac{W_{L S}}{G}$, callow degree of interference, it indicates how many olements of $X$ conflict for the same cache line (cf. section 3.1.2), and therefore it reflects quite well the degree of sclf-interforences occuring on $X$. Pinally, parameter $d=\frac{l_{n z}}{W_{13}}$ : called densily. corresponds to the average distance between two nom-zem clements on a row and on a column of original matrix if (cf. section 2). In other terms, it is a measure of the dregree of temporal and spatial locality of non-zero elements of matrix $A$, and consequently, of the references to arrity $X$.

Basing our analysis on the model obtained in section :3 and simulations, the role and importance of the above pairameters is discussed in the following subsections. A small subsection is also devoted to discussing the difference between direct-mapped and set-associative caches. For silk. of simplicity, the experiments used to make the graphos of this section are mainly based on uniformly distributed mie trices, but account quite well for phenomenons oceuring in real sparse matrices.

\subsection{Line size $L_{S}$}

Influence of $L_{S}$ on the intrinsic misses of $M$ atrix and Index The expression of the number of intrinsic misses (3) shows that this number decreases hyporholically witl $L_{S}$ (cf. figure 13). Therefore, a small increase of $L_{S}$ bring important reductions of the number of intriusice misser.

Influence of $L_{S}$ on self and cross-interference misses Let us assume that $\frac{W_{B}}{C_{s}}>1$. In the case where non-zero elements are uniformly distributed across the sparse matrix. the approximate number of self-interference misses is giwn by (1). This expression is a function of $l$,

$$
N_{c m}^{\text {self }}=\alpha-\frac{\beta}{L_{S}}
$$

Therefore, $N_{\mathrm{cm}}^{\text {self }}$ grows with $L_{S}$, though this incrcase is more or less moderate depending on $\beta$. Otherwise, $N_{c m}^{\text {cross }}=$ $\frac{2 N_{n,}^{2} \times L_{s}}{C_{S}}$, therefore the number of cross-interference missis grows linearly with $L_{S}$. Consequently, an incroas: on $l_{s}$ 


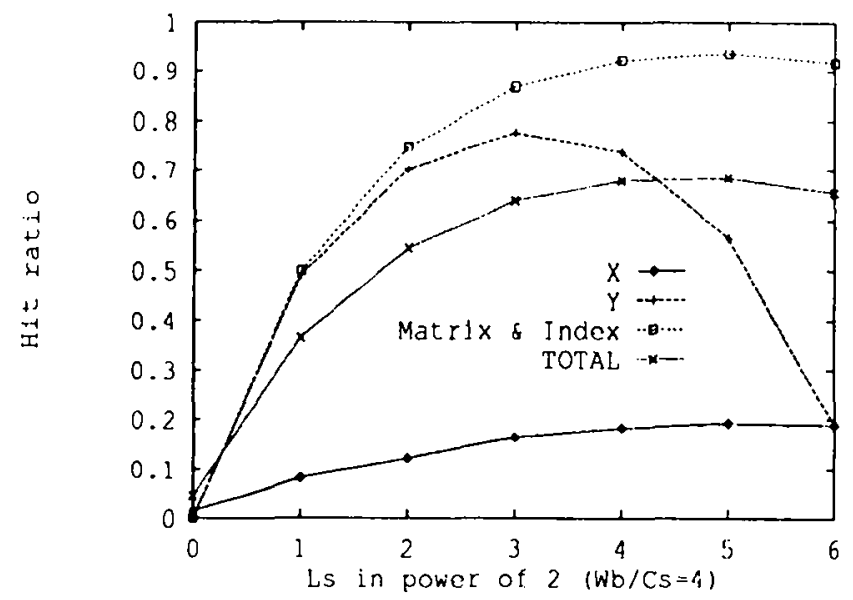

Higure 13: Influence of $L_{S}$ on the total hit ratio and the hit ratio of each array.

corresponds to an increase on both the number of selfinterference and cross-interference misses (cf. figure 14).

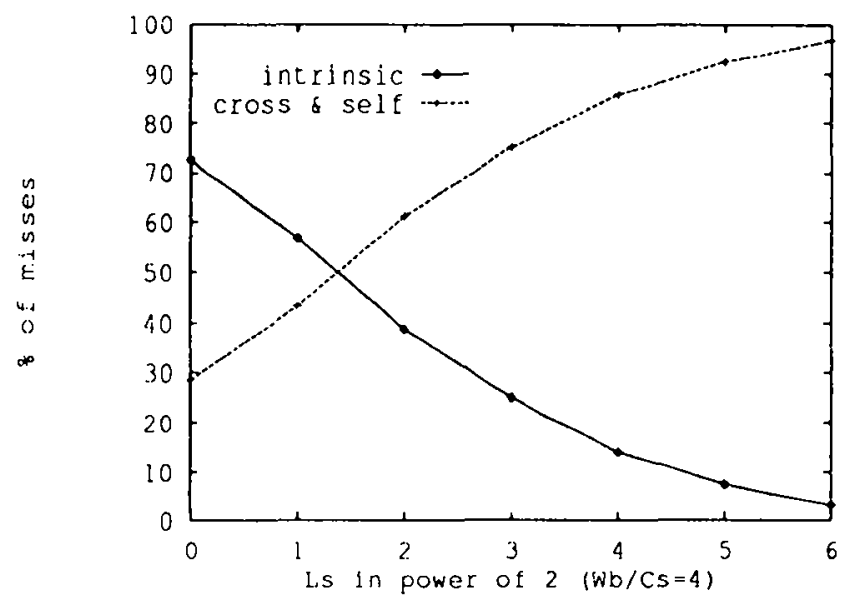

Figure 14: \% of intrinsic misses and self + cross-interference misses for different valucs of $L_{S}$.

The consequence of the previous observations is that the hit ratio of all arrays but $X$ increases very quickly (hyperbolically) with $L_{S}$. On the other hand, because of cross and self-interference misses, the hit ratio of $X$ increases much more moderately (or somctimes even decreases) when $L_{S}$ grows.

Therefore, for small values of $L_{S}$ the main cause of cache misses are arrays Matrix and Index, while for high values of $L_{S}, X$ accounts for the major part of cache misses (cf. figure 15).

Consequently, devoting important efforts to benefit from the temporal locality of $X$ should be considered only when $L_{S}$ is such that $X$ becomes a major cause of cache misses, otherwisc little improvernents of total hit ratio can be expected (cf. figure 15).

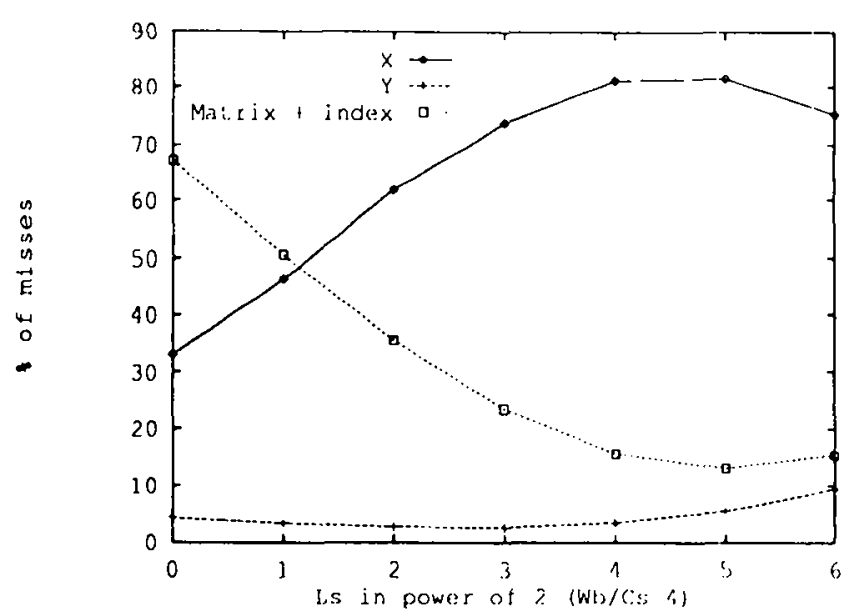

Figure 15: \% of misses of each array for different valucs of $L_{S}$.

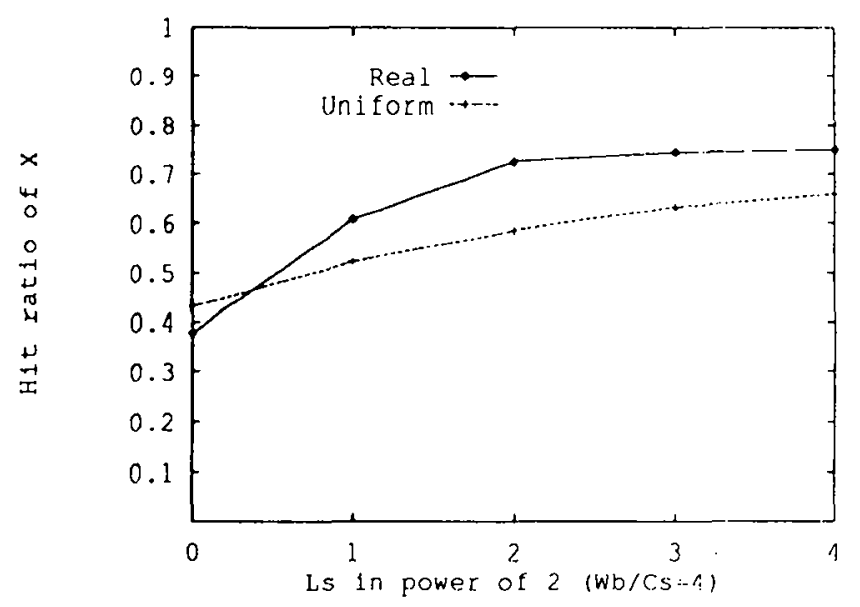

Figure 16: Influence of the distribution of non-sero elcments on the spatial locality of references to $X$

Proper values of $L_{S}$ for finite-element sparse matrices Due to the properties of mesh structures (where each node has a relatively constant number of neighbors) and the use of renumbering algorithms to minimize bandwidth. finite-element sparse matrices exhibit a non-uniform distribution of non-zero elements. They are grouped by packs of 2,3 or 4 elements depending on the mesh type. This spatial locality of non-zero elements induces a spatial locality of references to array $X$. Therefore, $L_{S}=2$ or 4 is sufficicut. to make use of this locality, while little improvement (atl be expected for higher values of $L_{S}$.

As it can be seen on figure 16 , the reduction of cache misses between $L_{S}=1$ and $L_{S}=2$ is impressive, while it steps down after $L_{S}=4$. 'This phenomenon is characteristic of finite-element matrices. It can be noted on figure 16 that an increase of $L_{S}$ breeds progressive instead of drastic. improvements on the hit ratio of $X$, when the non-zero olements are uniformly distributed within the sparse matrix. The shape itself of finite-element sparse matrices (cf. fig- 
ure 6) suggests a greater spatial and temporal locality than of uniformly distributed matrices. Ilowever, both uniform and finite-element distributions tend to behave similarly for large enough cache line sizes, i.e when the locality effect of finite-element sparse matrices does not show anymore.

\subsection{Degree of interference $w=\frac{W_{S}}{C_{S}}$}

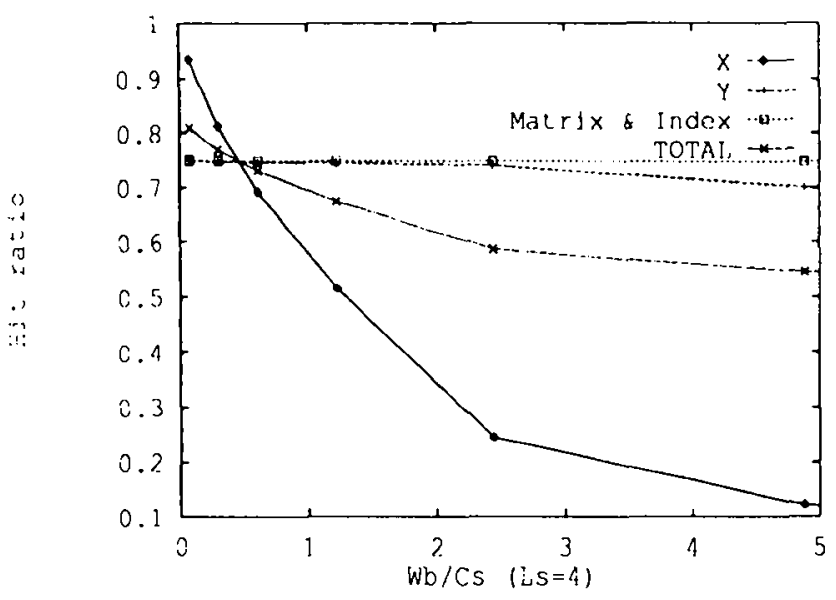

Figure 17: Influcnce of $\frac{W_{B}}{C S}$ on the total hit ratio and the hat ratio of each array.

Solf-interforence misses 'The effect of $W_{B}$ and $C_{S}$ cannot be dissociated. For $W_{B}<C_{S}$, the number of selfinterference misses due to $X$ is equal to zero. This appears clearly when considering simplified problem $P^{\prime \prime}$ : the number of columns of $A^{\prime \prime}$, i.e the number of interfering columns, is equal to $\left\lfloor\frac{W_{B}}{C_{s}}\right\rfloor$ or $\left\lfloor\frac{W_{B}}{C_{S}}\right\rfloor+1$, that is, 0 or 1 . Therefore, once an element is loaded into a cache line, it cannot be flushed by another element. So, for $W_{B}<C_{S}$, the number of cache misses due to $X$ is optimum. Now, for $W_{I 3}>C_{S}$, model expression (1) shows that the number of self-interference misses increases hyperbolically with $w$

$$
N_{c m}^{s \in l f}=\alpha-\frac{\gamma}{u}
$$

Now, if the previous expression is considered as a function of $w$, and is differentiated it, then it appears that the increase of $N_{\mathrm{cm}}^{\mathrm{elf}}$ becomes small (i.c less than $10 \%$ ) whenever $w \geq$ $\sqrt{10 \alpha}$.

So, thrce cases can occur. First $w<1$, the number of self-interference misses is negligeable and it is not useful to reduce $W_{B}$. Second $w \simeq 1$, in this interval the number of self-interference Inisses increases hyperbolically with $w$, and therefore very significant improvements can be obtained through slight bandwidth reduction. Third $w \gg 1$, and the number of solf-interference misses is close to maximum (nearly no element of $X$ is reused), and only a drastic bandwidth reduction may bring improvements.

Cross-interference misses When $w \gg 1$ is sufficiently large, because of self-interferences only, there is little reuse on $X$. Therefore, the effect of Matrix and Index, i.c cersisinterferences, can only be redundant with that of $X$.

When $w \leq 1$, there are no self-interference misses. In that case, alive (i.e currently used) elements of $\lambda$ are located in an area of size $W_{B}$ within cache. Now, as mentioned in section 3.2.2 Matrix and Index can be considered as twe "trains" of references moving across the cache. Therefore, the larger $W_{B}$, the higher the probability that these "trains" mect the area of alive elements of $X$, i.e the higher the probability of cross-interferences. Still, these cross-interference misses account for a relatively small share of total misses, unless $L_{S}$ is large, ie there are few inlrinsie messes (ce. ligeure 18).

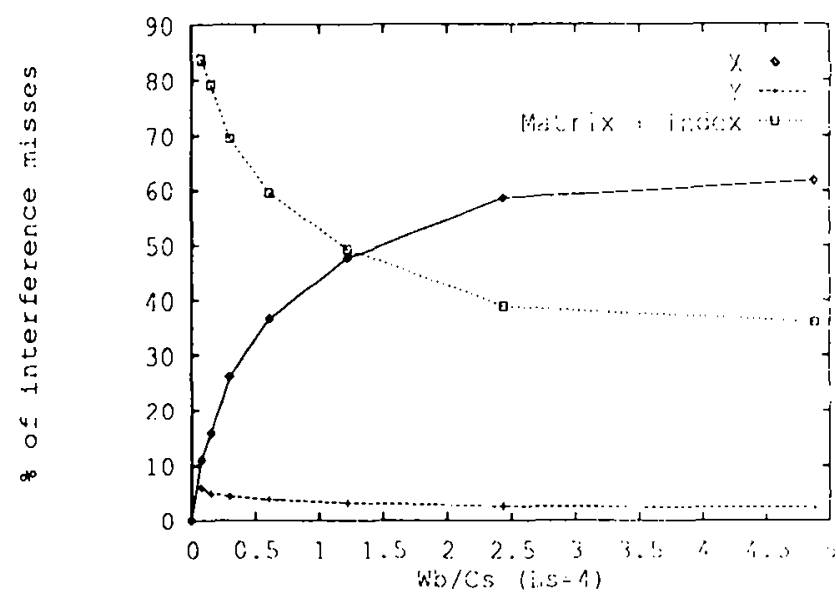

Figure 18: \% of interfercuce messes for dellrerml raluses of $\frac{W_{H}}{C_{S}}$.

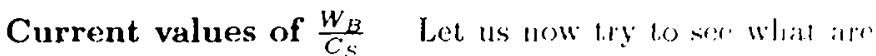
the values of $\frac{W_{B}}{C}$ currently found

The size of numerical problems tends to grow, and cour. sequently $W_{B}$ grows accordingly. When banduadlh reduction renumbering algorithms are employed. $H_{B} \simeq \sqrt{V} \mathrm{or}$ $W_{B} \simeq N^{\frac{2}{3}}$ or $W_{B} \simeq \frac{N}{10}$ according to the problem y.j. while $W_{B} \simeq N$ when minimum-degrec remumbering alud rithms are used [7]. Large current problem sizes lange fronu $N=10^{5}$ to $N=10^{6}$, therefore $W_{B}$ generally varies bectweren $W_{B}=900$ and $W_{B}=10^{6}[7]$.

For single-cache machines, $C_{S}$ clearly tends to grow. $C$. values of more than 256 Kbytes can now be found [1i]. So, if cache sizes increase fast enough $\frac{w_{y}}{C_{4}}$ will soon br on the "safe zone" (i.e $\frac{W_{B}}{C_{S}} \ll 1$; 256 Kbytes cache. $N=10^{\circ}$. $W_{B}=N^{\frac{2}{3}}, \frac{W_{B}}{C_{s}}=0.01$ ). On single-cache machines equipped

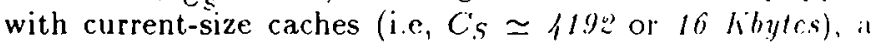
large third-dimensional problem (i.e, $N \simeq 10^{5}, 11_{13} \simeq . \div$ to $W_{B}=N$ ) exhibits a $\frac{W_{B}}{C s}$ ratio of 0.51 .0 .5 .

The increasing popularity of multi-level caches mation small (primary) caches more frequent. The size of such caches is currently of the order of / Kibytes [1i]. What is more, $N$ also tends to grow, and minimum degree is a ratlec popular algorithm (cf. [11]). So very large matios $\frac{W_{L S}}{i: 3}$ m! 
become more frequent ( $/$ kbytes cache, $N=10^{5}, W_{13}=N$, $\left.\frac{W_{u}}{C_{s}}=100\right)$.

\subsection{Density $d=\frac{n_{n z}}{W_{B}}$}

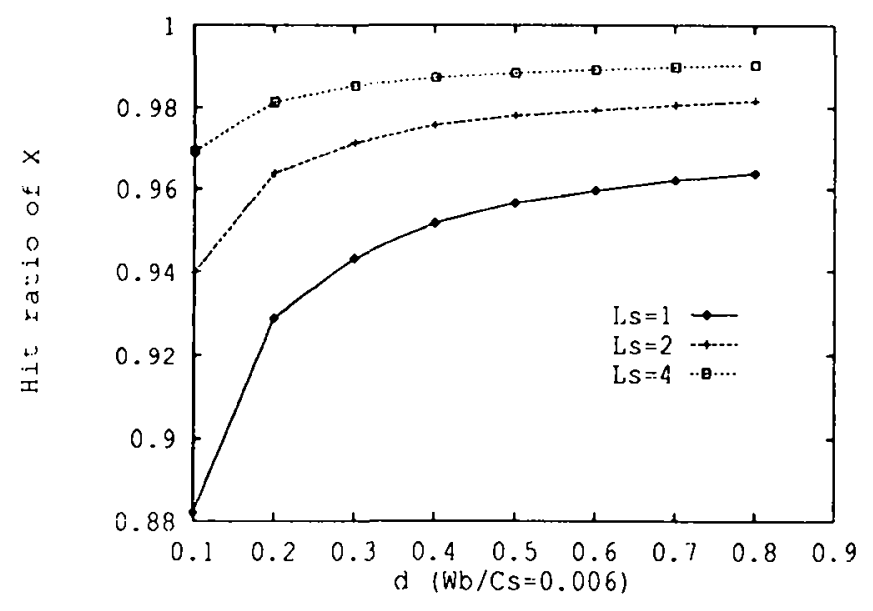

Figure 19: Influence of the density on the hit ratio of $X$ for $\frac{W_{Q}}{C_{S}}<1$.

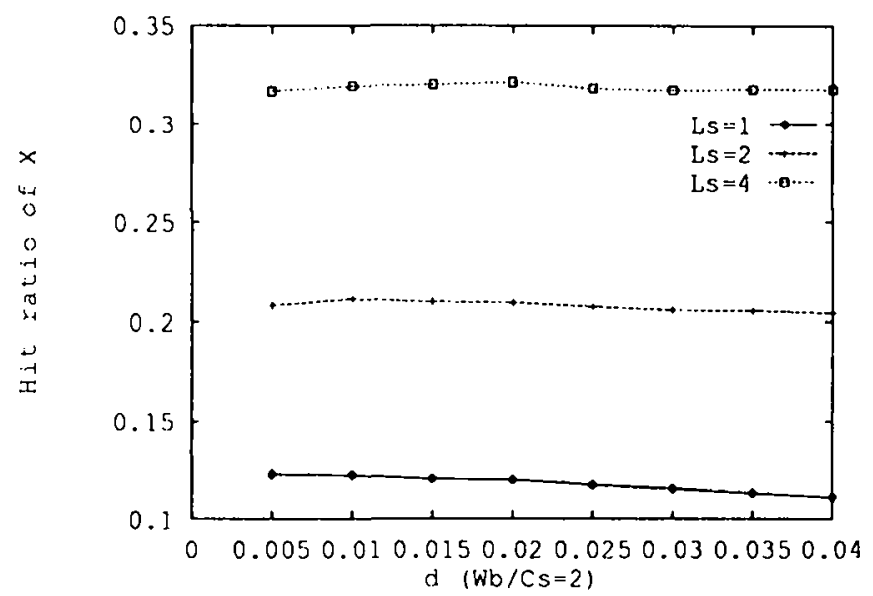

Figure 20: Influence of the density on the hit ratio of $X$ for $\frac{W_{B}}{C_{S}}>1$.

Let us now consider parameter $d=\frac{n_{n s}}{W_{B}}$. $d$ can be considered as the "density" of non-zcro elements on one row of matrix $A$. When $d$ is very high matrix $A$ looks very much like a banded dense matrix, while the matrix is "very sparse" when $d$ is relatively small.

Depending on $w$, the density of non-zero elements induces two different phenomenons.

If $u<1$, the smaller $W_{B}$ (i.e the larger $d$ ) the less crossinterferences occur (cf. paragraph 4.2). Consequently, it is possible to benefit from the temporal locality of references to $X$ (cf. graph $L_{S}=1$ of figure 19). For the same reason, it is also possible to benefit from spatial locality. Indeed, once an clement of $X$ is loaded $L_{S}-1$ consecutive elements of the same array are also loaded. Though they are not im- mediately refcrenced, they are not. flushed from cache (as seen above), and therefore they stay into cache until thry are referenced. That is why array $X$ also benefit.s from spattial locality in this case, independently of the distribution of non-zero elements (cf. paragraph 4.1 aud figure 19).

Now it can be observed that, for a fixed value of $W_{13}$, when $n_{n z}$ is large there exists an important potential reuse on $X$. Since it is possible to benefit from temporal locality when $w<1$, the higher $n_{n z}$ (i.e the larger $d$ ) the higher the hit ratio of $X$ (cf. figure 19). Neverthcless, a high value of $n_{n z}$ slightly worsens cache pollution, though $n_{n z}$ rnust be quite large for this phenomenon to comnterbalance the benefits from spatial and temporal locality (cf. figure 19).

When $w>1$, the behavior of $X$ is not correlated to $d$ anymore because interferences (cross and sclf) occur so often that benefiting from temporal and spatial locality becomes hypothetical (cf. figure 20).

Usual values of $d$ For 2-dimensional finite-element prohlems, the average number of non-zero elements per row is of the order of 10 , while it is of the order of $100 \mathrm{f}(\mathrm{r}$ 3-dimensional problerns [7]. So essentially 3-dimensional problems are worth optimizing. As it has been seen in paragraph $4.2, W_{B}$ ranges from $\sqrt{N}$ (2-dimensional) or $N^{\frac{2}{3}}$ (3-dimensional) to $N$. Typically, the density of a :3. dimensional problem may range from 0.001 to 1

\subsection{Set-associative caches}

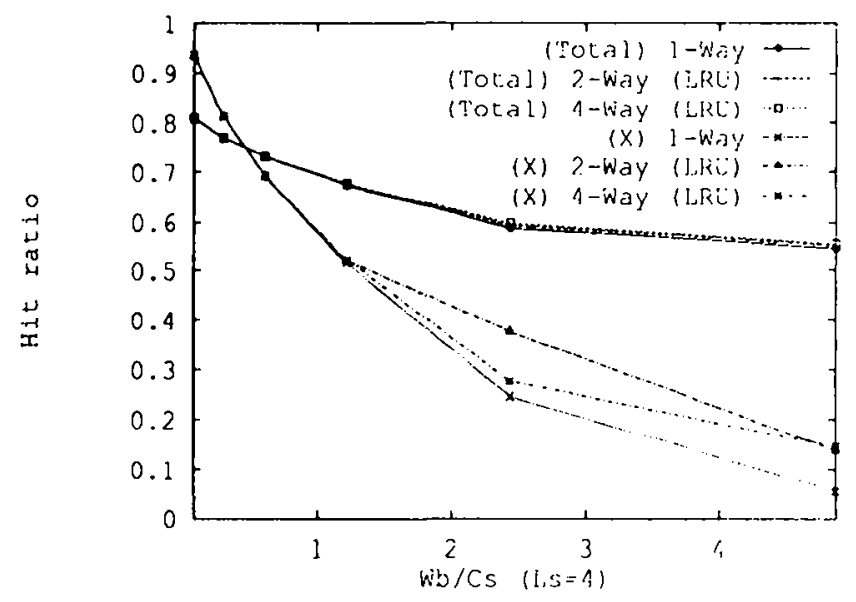

Figure 21: Performance comparison of set-associatime and direct-mapped caches for different values of $u$

Though the model presented in section 3 corresponds to direct-mapped caches, it can be extended to set-associative caches (cf. section A). Nevertheless, simulations can already show that associativity brings little improvements on total hit ratio, and more particularly on the hit ratio of $X$. Basically associativity is helpful when interferences occur. Now, when $w<1$, there are very few interferences, and when $w>1$, interferences are so numerous (at least $u$ elements of $X$ conflict for the same cache location) that at 
2-way or 4-way associalivity brings litile or no improvement. (cf. figure 21 ).

\subsection{Synthesizing the analysis}

In most applications there is a tradeoff related to $L_{S}$. When $l_{S}$ is too large, cache pollution becomes important and threatens further gains. For $\mathrm{SpMxV}$ again, the tradeoff exists. Increasing $L_{S}$ worsens cross and self-interferences. What is more it has been noted that only small line sizes (typically $L_{S}=2$ or 4) are necessary to take into account the particular locality of finile-clement matrices. However, in SpMxV, intrinsic misses account for the major part of cache misses, and therefore even very large line sizes do bring substantial improvements. However, the reduction is hyperbolic, and therefore there is again a threshold beyond which little improvements can be expected (typically $L_{S} \geq 16$ ). Anyway, as long as intrinsic misses account for a large fraction of total misses, i.e as long as $L_{S}$ is small (lypically, $L_{s} \leq 2$ ), decreasing the miss ratio of $X$ does not bring significant improvements on the total hit ratio.

'The degree of interference $w=\frac{W_{B}}{C_{S}}$ strongly influences the hit ratio of $X$. When $w<\overline{1}$, there are no selfinterference misses, and therefore high total hit ratio can he achieved if $L_{S}$ is large. If $L_{S}$ is small (typically $L_{S} \leq 4$ ), the percentage of cross-interference misses is far smaller than intrinsic misses, though may significantly increase for large values of $L_{S}$. Reducing $W_{B}$ also decreases the number of cross-interferences. When $w \simeq 1$, the number of selfinterferences grows hyperbolically with $w$, and therefore significant gains on the hit ratio of $X$ can be expected through bandwidth reduction. When $w \gg 1$ (typically $w \geq 10$ ), the number of self and cross interferences are redundant and close to maximum. If $I_{S}$ is large, misses of $X$ account for the major part of total misses.

When $w<1$, the higher the density of non-zero elements $d=\frac{n_{n x}}{W_{x}}$, the better the hit ratio of $X$. When $w>1$, it is hardly possible to make use of the locality of $X$, and therefore the density does not influence much the hit ratio of $X$.

Finally, simulations tend to show that, in most cases, associativity brings little improvements on the hit ratio of $X$ and the total hit ratio.

\section{Improving the behavior of Sp- $\mathrm{MxV}$}

In this section, possible software and hardware optimizations are discussed. Two different approaches for software optimization of $\mathrm{SpMxV}$ are distinguished: an algorithmic approach which aims at reducing bandwidth using renumbering algorithms, and a software approach based on particular blocking techniques. For hardware optimizations, the ways to reduce misses corresponding to regular and nonregular references are considered.

\subsection{Software optimization: Bandwidll reduc- tion.}

Two main kinds of remumbering algorithurts are enployed: bandwidlh reduction algorithms which are derivatives of that of Cuthill and McKee, and the mimmum-degree algorithm [11]. According to George [11], reducing bandwidlh is not closely related to minimizing arithmelic operations and storage. On the other hand, minimum-degree algorithm is efficient for finding low-fill orderings. Therefore, this second renumbering scherne tends to become popular. llowever, it must be noted that an effect of minimum degree is to scatter non-zero elements across the natrix (cf. figure 22), white bandwidth reduction algorithms are generally very eflicient. in grouping non-zero elements (c.f. figure 22). So, if $\mathrm{mlm}$ mum degrec is more efficient for LU factorization as shown by George, it is far lesss profitable for $S p M \times V$ in terms of locality, because it widens considerably matrix bundwillith.

Both LU factorization and $\mathrm{SpMxV}$ often occur in the same program. Since LU factorization is far more costly then $\operatorname{SpMxV}$, optimizing renumbering for this operation would be reasonnable (i.e sacrificing bandwidth). Howerer, for a given matrix, $S_{p} M x V$ is often executed several times. vector $X$ being the only data changed on eatch iteration. Therefore, it is after all profitable to mate a copy of $1 . \mathrm{h}$ : matrix and renumber it for SpMxV only, since the cost of the renumbering process is not prohibitive (of 1.he order of one execution of SpMxV). It should also be kejt, in miml. that renumbering in order to minimize bandwidth is profitable in our case, only if the degres: of interference w: call be made smaller than 1 .

\subsection{Software optimization: Blocking}

\subsubsection{Classic blocking}

The reason why sparse codes do not work on caches winn matrix parameters are much larger than cacle paramelens is the same as for dense codes: because elements to be reusial cannot be kept in cache. However, a solution valid for dense codes [5], i.e blocking, is not valid for sparse codes, because each element is not reused a sufficient number of times 10 override the overhead of blocking

Moreover, sparse codes exhibit an irregular localily which cannot be forseen at compile-time (i.c a priori), while densi codes exhibit regular locality, which can be exploited it. compile-time. If classic blocking techniques were to bo used for $\mathrm{SpMxV}$, part of its irregular localuty would probialily lw lost, and large overhead data would be added.

Let us try to explain and formalize these latter notions. Consider original matrix $A$ is blocked horizontally and wertically, using rectangular blocks. One block has $B_{Y}$ rows and $B_{X}$ columns. Therefore, if each block is stored rowwise, $B_{Y}$ integers must be stored indicating the number of non-zero elements on each row. $B_{Y}$ rows of the matrix are divided into $\frac{W_{B}}{B_{X}}$ verical blocks. Since, there are $\frac{N}{B_{y}}$ blocks of size $B_{Y}, \frac{N \times W_{B}}{B_{Y} \times B_{X}}$ integer elements must be stored for this blocked algorithm (while only $N$ elements are needed for the original non-blocked algorithm).

Now, there are two ways to execute this blocked Sp.Hxl: row-wise or column-wise. If $\mathrm{SpMxV}$ is executed columm- 

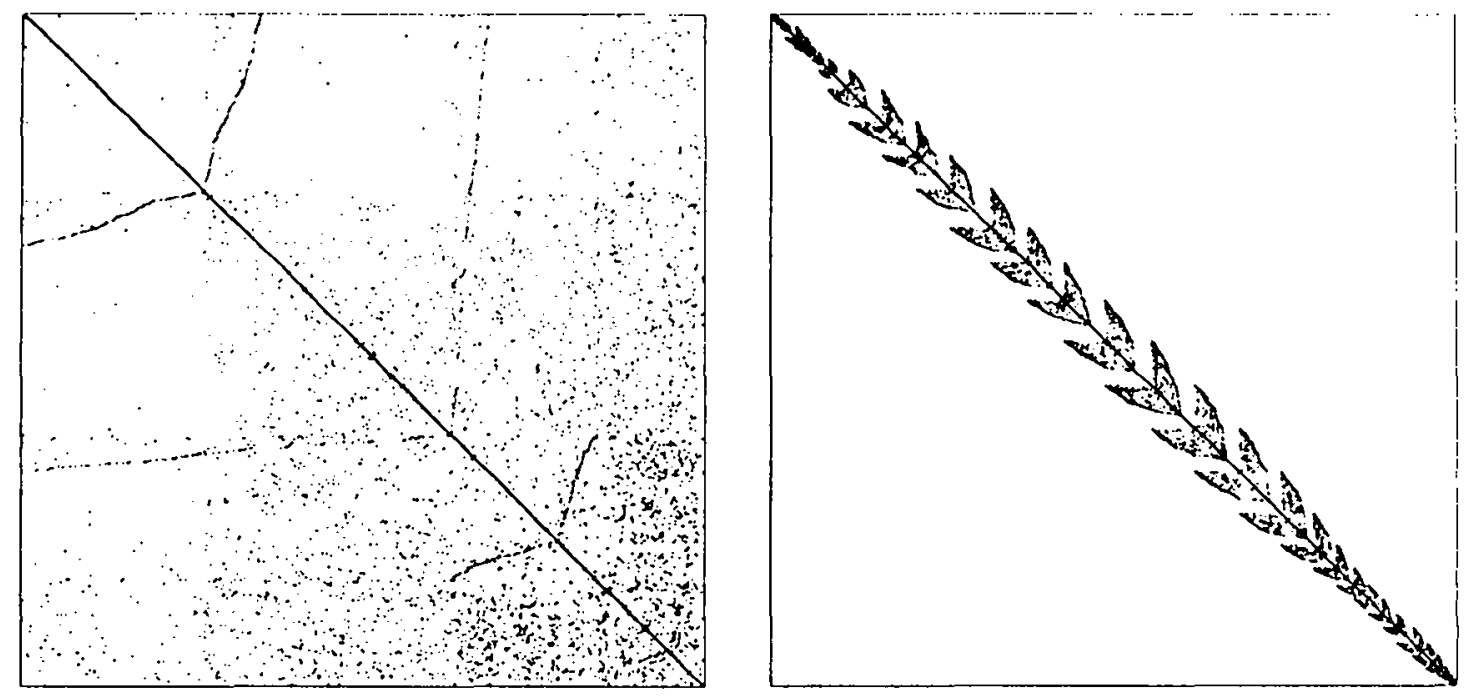

Figure 22: Matrix BCSPWROg of the Harwell-Boeing suite after application of minimum degrec algorithm and altiel application of a bandwidth reduction algorithm.

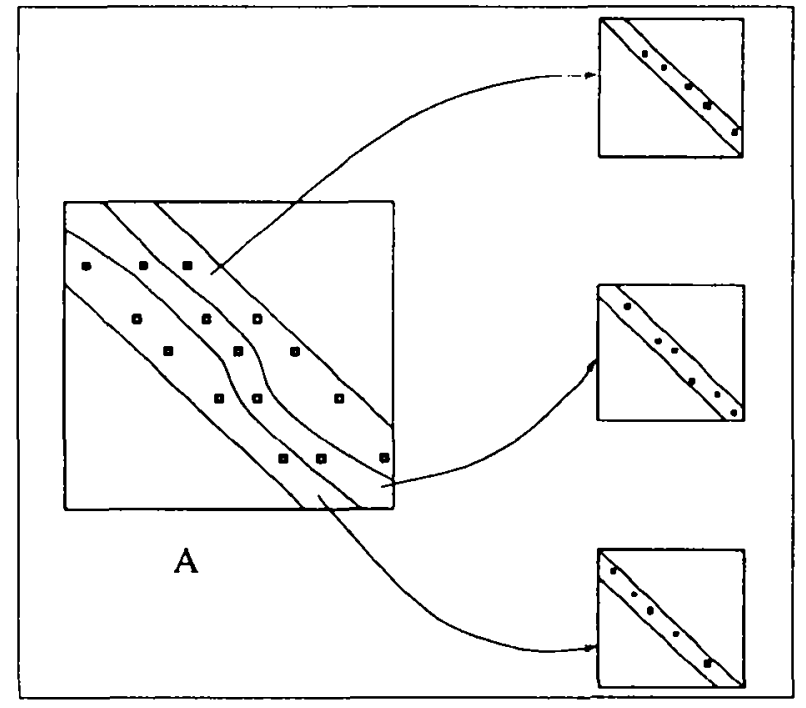

ligure 23: Splitting a sparse matrix with a large band into several submatrices with a small band.

wise, then clements of $Y$ probably cannot be kept into cache from one column to another. Consequently, the number of cache misses due to $Y$ is multiplied by $\frac{N}{B_{x}}$. If $\operatorname{SpMxV}$ is executed row-wise, then the reuse of $X$ is degraded in turn. However, since $X$ does not exhibit the same flawless spatial and temporal locality as $Y$, then it is preferable to degrade the reuse of $X$ rather than that of $Y$, i.e to execute $\mathrm{SpMxV}$ row-wise.

Since on one column of a block $B_{X} \times B_{Y}$ there are averagely $d \times B_{Y}$ non-zero elements (where $d=\frac{n_{n x}}{W_{B}}$ is the density of the sparse matrix), then in the best case, the miss ratio of $X$ can be divided by a factor of $d \times B_{Y}$. In order to have at least 2 non-zero elements per column of a block (i.e that an element of $X$ is reused at least twice per block), it is necessary to have $\frac{n_{n x} B_{Y}}{W_{B}} \geq 2$ for blocking to be profitable. Since at most $B_{Y} \stackrel{{ }^{B}}{=} C_{S}$, the minimal condition for benefiting from rectangular blocking is $\frac{n_{n a} C_{s}}{W_{B}} \geq 2$.
Actually, because of the $\frac{N^{2}}{B_{Y} \times B_{X}}$ new intrinsic misses, it is necessary to have $\frac{n_{m s} C_{s}}{W_{B}} \gg 2$ in order to get anly impreve:ment.

Therefore, in most cases classic blocking of sparse miltrices degrades the hit ratio of $Y$, and brings littic improvements on that of $X$ (cf. figure 25 ; the optimal block sizes were found experimentally: $C_{S}=102 /, H_{X}=100$. $B_{Y} \simeq 25$ ). Moreover, significant overhead data worsiens th: total hit ratio by addition of intrinsic misses (cf. ligure 25). Only if the matrix is nearly dense $(d \simeq 1)$, classic blocking is profitable due to the importart potential reuse per element of $X$.

\subsubsection{Blocking by diagonal}

It is clear that any software optimization techniques should take into account the specifics of $S p M \times V$. First of all, in opposition to most dense codes, it must be assimilated that. one computation corresponds to one non-zero element and not to any element of the matrix. Therefore, any blocking technique should implicitly deal with blocks of non-scto elements rather than blocks of matrix elements.

Second of all, in dense matrices, the elements of symmetry are columns and rows, which explain why such matrices are blocked using rectangular shapes. The elements of symmetry of banded sparse matrices are diagonals. "Therefort. it seems natural to block along diagonals, otherwise too many blocks would be half-empty and would degrade llw efficiency of the blocking techinique.

According to section 4.2, the original large hand sloulal be split into several small bands, such that their widtl is of the order of cache size ( $\mathrm{cf}$. figure 23). Now, these blocks should be based on non-zero elements and not on arbitrary geometric dissection. Moreover, having the number of nonzero elements on each of the different diagonals constaut. (except for the first and last diagonal blocks) would reduce: the overhead data to be kept, as seen in above paragraph.

For that purpose, $n_{n z}$ the average number of non-zero clements per row and the number of non-zero element.s on each 


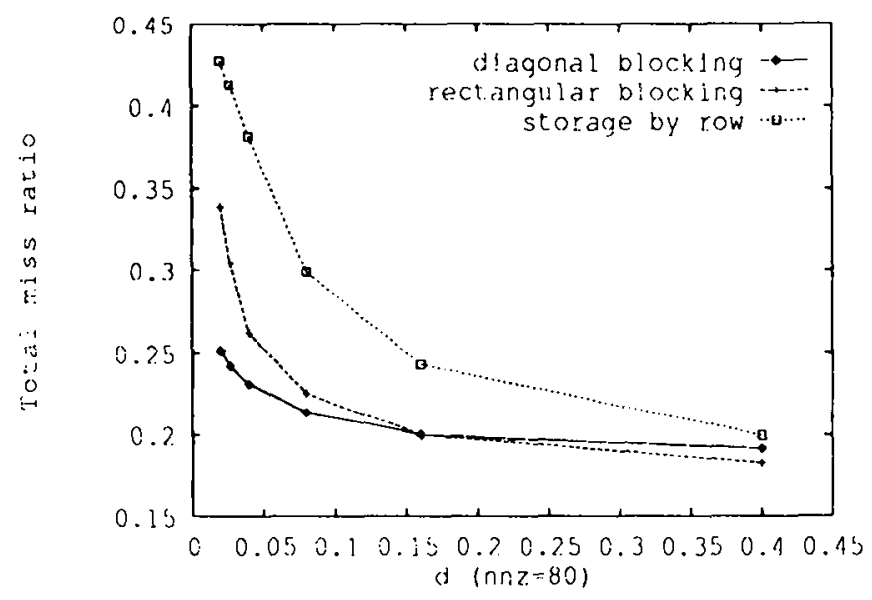

Figure 24: Effect of diagonal blocking on the total miss ralow.

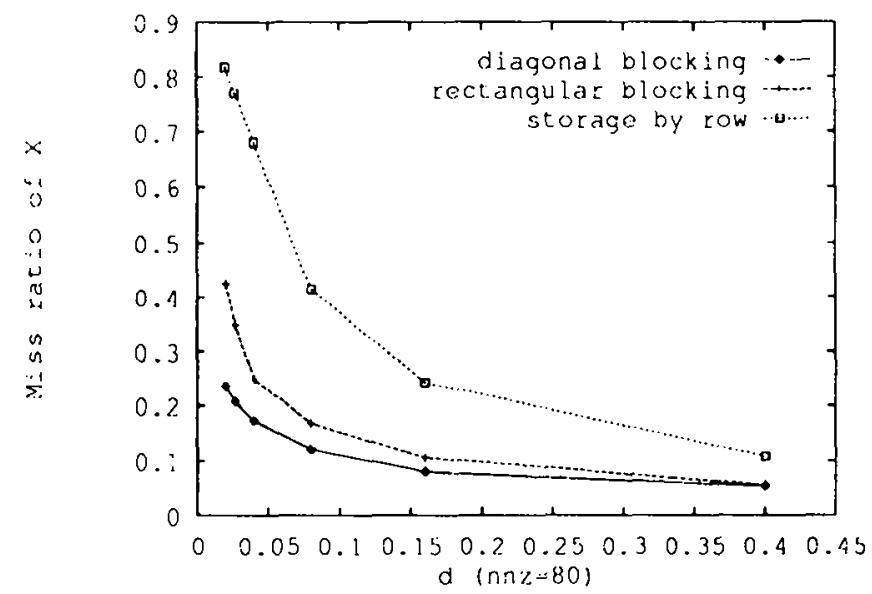

ligure 25: Effect of diagonal blocking on the miss ratio of $X$.

diagonal of the original matrix must be computed. Since the goal of the method is to obtain a collection of banded matrices which bandwith is smaller than cache size, the original matrix needs to be split into approximately $n_{B}=\left\lceil\frac{W_{\Omega}}{C_{S}}\right\rceil$ submatrices. In all these submatrices except for the first and last one, the number of non-zero elements ought to be constant, approximately equal to $\frac{n_{n z}}{n_{B}}$. Additional data is necessary to store the number of non-zero elements on the rows of the first and last diagonal block. The corresponding code can be seen on figure 26 .

This blocking method is interesting only when $X$ is the major cause of cache misses, and the potential reuse is high (cf. sections 4.1 and 4.3). Though it increases the number of misses on $Y$ (they are nearly multiplied by $n_{B}$ ), it decreases the miss ratio of $X$, so that the improvement of the total hit ratio may be quite important (cf. figure 25 ). The main asset of the method is to be applicable even when the density is low, where other classic blocking methods would fail.

Moreover, the shape of certain types of matrices such as finite-element matrices suggests that diagonal blocking could be further enhanced. Inderel, in such matrices, there are generally three diagonals along which non-zero element.s are grouped (cf. figure 6). Therefore a first step to diagonal blocking would be to find the diagonals of "highest density" and block non-zero elements around them. Not, only, urarly. dense blocks of non-zero elements would be obtained, hut the number of blocks itself (and the overhead) would be: considerably reduced in many cases, since it would be lixid (and would not depend on $W_{B}$ and $C_{s}$ ).

However, all blocking techuiques inliroduce bonnels of their own. In our case, the mumber of blocks deternine the maximum reuse per element of $X$. If there are $n_{s 3}$ blecks and $n_{n z}$ non-zero elements per row, then diagonal blocking

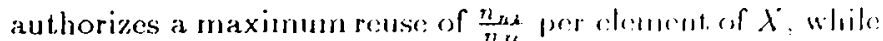
the theoretical maximum is $n_{n}$.

\subsubsection{Parallelization}

Rather than making use of data locality, supercomputers propose to speed up sparse computations by wetorizing arenses to data stored in compress formal. using latedwiar. Scatter/Gather [14]. For shared-memory multiprocessems with a memory herarchy, the issue is to parallelize sparse: codes while optimizing load balancing and communtiontions between global and local memories. Actually, litule inlormattion is known on the behavior of such architectures under sparse workloads. Saad and al. [20, 21] proposed a ben.hmark suite for evaluating the performance of sparse rode. and experimentally analyzing their locality and inluerent parallelism. Yang [26] presented a set of parallelized spause: primitives, where parallelism is extracted at the algorithmic: level. Davis [1] also proposed a parallel implementation of sparse Gaussian elimination based on an algorithunic trials. formation, and studied slightly the impact of his sehrum: , communications between global and local momories.

However, little effort has been devoted to allalyous and modeling the impact of parallelization on the beliavior of sparse codes on a memory hierarchy. Now, for SpMxl. distributing tasks to each processor is equivalent to splitting original matrix into blocks of certain size and shape. Consequently, each subproblem assigned to a local cache is equivalent to original problem though with smaller fatrameters, and therefore, the whole previous analysis can be. applied to each subproblem separately.

This property can be used to detcrmine tle impact of blocking on communications. Actually, it may appear thal the most natural and most commonly employed methods for blocking sparse matrices (e.g. blocking by rows) may nol be the best regarding communications between the diffirent levels of memory. For instance, the original storagc-byrow scheme already allows an easy parallelization of $S_{p} M x V$ (cf. figure 5) on loop $I$. However, analysis of section 4 shows that a large bandwidth breeds an important miss ratio of $X$. Now, if SpMxV is blocked on loop $I$ and the bandwith of original matrix is large, then the bandwidth of the pr. sulting submatrices remains the same. Therefore, the mise ratio of $X$ on each local cache may be high.

On the other hand, blocking by diagonal laas several assels which can be profitable to parallelization and locality also, especially by improving data reuse without hringing significant overheads. Since the original problem (a bauded sparse: 


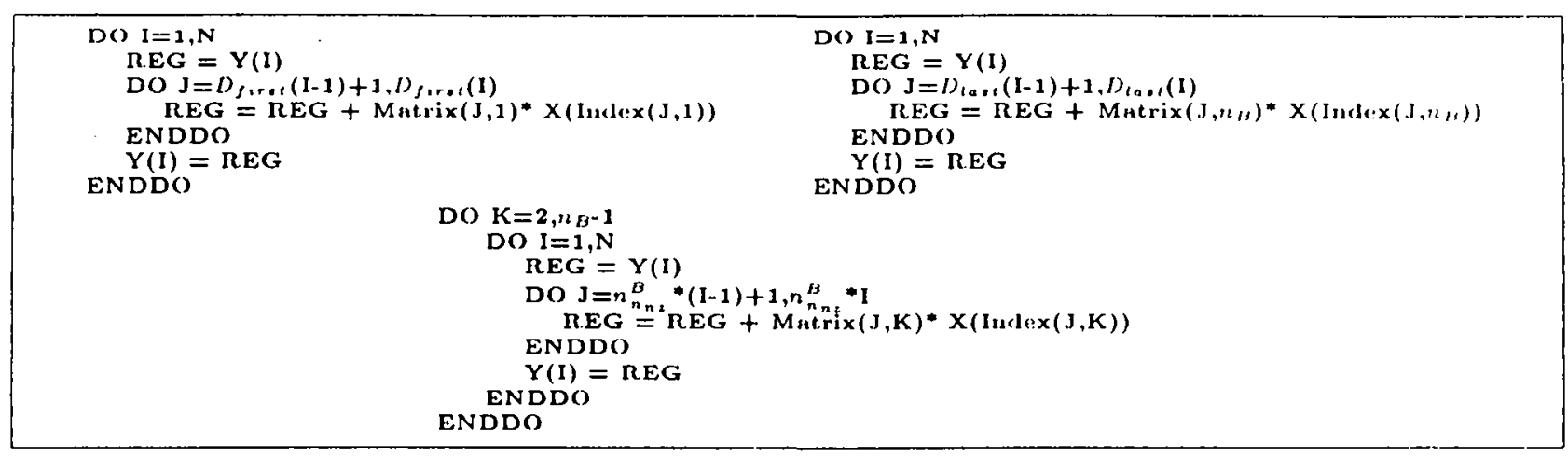

Figure 26: Blocking by diagonal.

matrix) is split diagonally, the submatrices bandwidth is smaller than that of original matrix (actually it is equal to that of original matrix divided by the number of blocks). 'Therefore, on each local cache the miss ratio of $X$ may be far smaller than when blocking by row. Consequently, parallelizing this way maturally decreases the burden on local aches by reducing the bandwidth, and consequently the litt. ratio

Another asset of this blocking method is that, once the original problem has been divided into subproblems, it is possible to apply any storage method for these subproblems, not inevitably storage by row. Now, jagged diagonal [3] and generalized columns [9] are among the most efficient storage lechniques for vector execution. 'Therefore, if a tradeoff between parallelization, vectorization and data locality must be: found, blocking by diagonal may ensure non negligeable data locality while still authorizing vectorization (on each subproblem separately)

\subsection{Hardware optimizations}

\subsubsection{Regular references}

In a number of cases, intrinsic misses account for the major part of cache misses (cf. section 4.5). These misses are due to arrays exhibiting flawless spatial locality and deprived of t.cmporal locality. Some processors are now equipped with pipelined bypass [13] which are well suited for loading arrays with high spatial locality, and which also avoid cache pollution with arrays deprived of temporal locality by not storing them into cache. Otherwise, this problem can also lue solved with many techniques already proposed for regular codes. Large line sizes [23] provide a simple solution for reducing the impact of such misses. Jouppi also presented a Multi-Way Streamed Buffer [15] which aims at removing completely intrinsic misses. Inspite of all, whenever intrinsic misses account for a minor part of total cache misses, self-interference misses due to array $X$ bccome the main issue.

\subsubsection{Irregular references}

little solutions have been implemented in the industry for dealing with sparse codes. The most classic and efficient one is the scatter-gather implemented on many vector supercomputers [14]. However, this feature aims at vectorizing non-regular accesses to incmory rather than optimizing the reuse of elements accessed. Other original solutions have been proposed, but up to now few resulted in actural machincs or at least few published results for the: mommon. Seznec and al. [22] propose an interconnection network t. lat. would behave efficiently under sparse code workloads. Ible:t and al. [12] intend to implement the Edimburgh Sinurse Pro. ccssor, an architecture dedicated to finite-clcment problems. Wolfe and al. [25] work on a coprocessor The While Inar. implementing specific sparse primitives. Finally, Amans, and al. [2] presents the Sparse Matris: Solming Marhine which is a parallel computer using a ruemory design arlatefor to sparse computations.

Most of the previous projects have not yet hren inuplemented or at least few performance resultis have lesen ful,lished. Actually, the majority of these projects concern doclicated machines rather than solutions for optimizing and adaptating current memory hierarchies to sjarso commutations. Let us consider which design would be the mosi convenient for these applications. When $w \gg 1$, interferences are so numerous that exploiting locality withiu Sp$\mathrm{MxV}$ becomes impossible in a usual cache (cf. section 4.5). However, if it were possible to determine placement of data within cache (as in local memory), many interforences would be avoided. First, only one memory location is actually required for arrays $D, Y$, Index and $M$ alrit and the rest can be devoted to array $X$ (thereby removilts cross-interferences). Second, the pattern of references to $X$ is known a priori, so if data replacement can be controlled (as in local memory), optimal replacenent could be achieved, as shown in [4] (thereby mimimining sellinterference misses). On the other liand, wen $u<1$, callw: makes a very good job at exploiting spatial ambl temporal locality without any optimization (i.e no overhead) reyuired (cf. section 4.5). So depending on problem parancters Sp$\mathrm{MxV}$ would require the upper-level memory ti.) have the properties of a local memory or of a cache, in olluer l.1.rul. of a hybrid memory system (cf. [6]).

\section{Conclusions}

This paper presents a methodology for modeling the irregular references of sparse codes using probabilistic methods. The model was shown to be very accurate for uniformly distributed matrices, finite-clement matrices renumbered with minimum degree algorithm, and still reflects quite woll tho 
behavior of finte-element matrices remumbered with bondwetlh reduction algorilhm.

The analysis of the model and simulations allowed to identify these main paramelers and their impact on the belawior of $S p: M \times V$. First of all, cache size and bandwidith are closely dependent. When bandwidth is smaller than cache, spatial and temporal locality of sparse matrices is well exploited and SpMxV needs not be optimized. Second, when bandwidth is greater than cache size, self and crossinterferences degrade the reuse of vector $X$ which cannot exploit its temporal and spatial locality. Moreover, in that case little optimizations can be expected if the line size is small because intrinsic misses are too numerous anyway. llowever, when line size is sufficiently large, then exploiting the potential locality of array $X$ may yicld significant improvements of the total hit ratio, especially in 3-dimensional finitc-clement problems where the potential reuse per element of $X$ is important.

Little hardware or software techniques exist for making use of locality within sparse problems. First, Bandwidh reduction renumbering algorithms may significantly improve the exploitation of locality within $\operatorname{SpMxV}$, but (he to I,U factorization minimum degree renumbering algorilhms (which widen considerably bandwidth) are generally preferred. Therefore, there is a tradeoff between the two algorithms which call actually be resolved through copying. second, blocking methods are considered. Classic rectangular blocking is proved to be efficient only when the matrix is nearly dense within its band, otherwise the method breeds too much overhead. A blocking technique blocking by diagonal that takes into account the specifics of sparse codes has been presented. It is shown to efficiently exploit locality where other blocking methods would fail, i.e when the matrix is "very sparse". Moreover, the parallel version of the: diagonally blocked algorithm on a multi-cache system would naturally reduce the burden on local caches.

\section{Acknowledgements}

We would like to thank J. Frhel and A. Seznec for their helpful comments and many enlightening discussions.

\section{References}

[1] S. G. Abraham and T. A. Davis: Blocking for parallel sparse linear syslem solvers, Proc. of Int. Conf. on l'aralled Processing, 1989

[2] 11. Amano, T. Yoshida and H. Aiso: $(S M)^{2}$ : Sparse matrix solving machine, Proc. of Int. Symp. on Computer Architecture, 1983

[3] F. Anderson and Y. Saad: Solving sparse triangular systems on parallel computers, Int. J. of High Speed Computing, 1 (1989), pp. 73-95.

[1] I. A. Belady: A study of replacement algorithms for a virtual-storage computer, IBM Systems Journal, Vol. 5, No. 2, pp. 78-101, 1966
[5] F. Bodin, (;. Fisentueis, 1). Windlociser. W. Jilly, a slralegy for array manaycment in local memory. Wavances in Languages and Compilers for Panallel Porcessing, Ml'T P'ress, 19)11

[6] (i. D). MeNiven and E. S. Davielson: Amalyses of memory referencing behavior for design of local memoris,

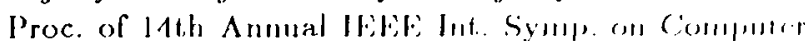
Architecture, $A C \mathrm{M}, 19 \mathrm{~s} \delta$

[7] 1. Duff, A. Erisman and J. K. Reid: Derect methods for Sparse matrices. Oxford Universily l'ress. Oxlord. England, 1987.

[8] I. Duff, R. Cirimes, J. Lewis: Sparse matrat lest probllems, ACM TOMS, 15) (1989), pp. 5is-(id

[9] J. Frhel: Sparse malrix mulliplacation on pecten computers, Int. J. of High Spered Computing, Vol. 2, No. 2. pp. $101-116,1990$

[10] C. Fricker, P. Robert: An andalylical cache motel, INRIA Report, INRIA Rocquesucourl, linaluce, July |egil

[11] A. George: Dircet solution of sparse positive defimb systems: some basic idcas and open problems. in Spars. Matrices and 'Their lises, edited ly l. S. Jnll. Andemic Press, 1981

[12] R. N. Ibbed, T. M. llophins and K. I. M. Mckinnrm: Architectural mechantsms to suplnort sparse lector pro-

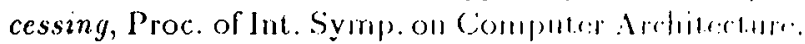
1389 .

[13] Intel Corporation: Intel i860 refcrence manual, l99)]

[14] J. G. Lewis and II. D. Simon: The impact of harduan Scatter/Gather on sparse Cianssian elominaluan. I'roe. of Int. Conf. on Parallel Processing, losij.

[15] Norman P. Jouppi: Improving cache teplacement hy Ih, addition of a small fully-associaluit cache and phi.fotrh buffers, IEEE, 1990.

[16] Monica S. Lam, Edward \&. Rothberg and Michanl E. Wolf: The cache performance and optimizations of blocked algorithms, Proc. 4th luternational conference on Architectural Support fo Programming langurand and Operating Systens, pp. 63-74, April 1991.

[17] D. A. Patterson and J. L. Ilennessy: Compulcr archltecture: a software approach, Morgin Kanfunamu Pullishers, Palo Alto, 1990.

[18] S. Przybylski, M. Horowitz and J. Hemessy: P'roformances tradeoffs in cache design. Proc of 151.h Amumal IEEE Int. Symp. on Computer Architecture, $\mathrm{A}(\mathrm{M}$. 1989.

[19] E. Rothberg and A. Gupta: Techniques for improring the performance of sparse matrix factorization on multiprocessor workstations, Proc of $\Lambda$ C M Siupereollputing 90.

[20] Y. Saad and H. A. G. Wijshoff: A benchmark packagr for sparse matrix computations. Proc of lit. (ont. an Supercomputing, 1988 
[21] Y. Siad and H. A. G. Wijshoff: SPARK: A benchmark package for sparse computalions, Proc. of Int. Conf. on Supcrcomputing, 1990.

[22] A. Scznec and Y. Jegou: Synchronizing processors through memory requests in a tightly coupled multiprocessor, Proc. of Int. Symp. on Computer Architecture, 1988.

[23] A. J. Smith: Cache memories, ACM Computing Surveys, vol. 14, pp. 473-530, September 1982.

[24] H.(Harry) A.(Arnold) G.(Gwendoline) Wijshoff: Implementing Sparse BLAS primitives on concurrent/veclor processors: a case sludy CSRD Report No. 843, Center for Supercomputing Research and Development, University of Illinois at UrbanaChampaign, January 1080.

[25] A. Wolfe, M. Breternitz Jr., C. Stephens, A. L. Ting, D. B. Kirk, R. I'. Bianchini Jr., J. P. Shen: The While Dwarf: A high-performance application-specific processor, Proc. of 14th Annual IEEE Int. Symp. on Computer Architecture, ACM, 1988.

[26] Ci. C. Yang: DSPACK: A parallel direct sparse matrix package for shared-memory multiprocessors, Proc. of Int. Conf. on Parallel Processing, 1990.

\section{A Appendix: Computing the number of cache misses due to array $X$}

\section{A.1 Methodology of resolution of the ba- sic problem}

II the following paragraphs, we indicate the method for computing the expression of $\pi_{\text {oud }}^{k}(i, j)$

State vector For cach element $k$ of $X^{\prime \prime}$, we define the state vector $\pi^{k}(i, j)$ as follows:

$$
\pi^{k}(i, j)=\left(\begin{array}{c}
\pi_{i n}^{k}(i, j) \\
\pi_{o u t}^{k}(i, j)
\end{array}\right)
$$

where $\pi_{i n}^{k}(i, j)$ is the probability that element $k$ of $X^{\prime \prime}$ is in the 1 -size cache on row $i$ of the computation, right before column $k$ is considered. And, $\pi_{\text {out }}^{k}(i, j)=1-\pi_{i n}^{k}(i, j)$.

Probability of transition let us now try to express $\pi^{k}(i, j+1)$ as a function of $\pi^{k}(i, j)$, that is, find a probability of transition matrix that could help describing this event.

Case $\mathrm{k}=\mathrm{j}: \pi^{j}(i, j)$ describes the probability of presence in cache of element $j$ of $X^{\prime \prime}$, on row $i$ of the computation, before column $j$ is considered. Now, when column $j$ is considered, element $j$ of $X^{\prime \prime}$ has a probability $p(i, j)$ of being referenced, that is a probability $p(i, j)$ of being loaded into the cache if it is not already there. This notion can be formalized by the following probability of transition matrix:

$$
p_{\text {load }}(i, j)=\operatorname{int}_{\text {out }}\left(\begin{array}{cc}
1 & p(i, j) \\
0 & 1-\mu(i, j)
\end{array}\right)
$$

Therefore, we can now write formally thal.

$$
\pi^{k}(i, j+1)=p_{\text {rrad }}(i, j) \times \pi^{k}(i, j)
$$

Case $k \neq j$ : if column $k \neq j$, then column $k$ has a probability $p(i, k)$ of breeding a reference to elcment $k$ of $X$, which would then flush element $j$ of $X$ from the cache. This notion can be formalized by the following probatility of transition matrix:

$$
P_{\text {flush }}(i, j)=\quad \operatorname{int}_{\text {out }}\left(\begin{array}{cc}
i n & \text { oul } \\
p(i, j) & 1
\end{array}\right)
$$

Transition from row $i-1$ to row $i$ : Thercfore, wi (:ill now express $\pi^{j}(i, j)$ as a funclion of $\pi^{j}(i-1, j)$.

$$
\begin{aligned}
& \pi^{j}(i, j)= \\
& \quad P_{\text {flush }}(i, j-1) \times \ldots \times P_{\text {flush }}(i, 1) \\
& \quad \times P_{\text {flush }}\left(i-1, n_{c}\right) \ldots \times P_{\text {Jlush }}(i-1, j+1) \\
& \quad \times P_{\text {load }}(i-1, j) \pi^{j}(i-1, j)
\end{aligned}
$$

Let us define $P_{(i-1, j) \rightarrow(i, j)}$ as follows

$$
\begin{aligned}
& P_{(i-1, j) \rightarrow(i, j)}= \\
& \quad P_{\text {flush }}(i, j-1) \times \ldots \times P_{\text {flush }}(i, 1) \\
& \times P_{\text {flush }}\left(i-1, n_{c}\right) \ldots \times P_{\text {flush }}(i-1, j+1) \\
& \times P_{\text {load }}(i-1, j)
\end{aligned}
$$

then $\pi^{j}(i, j)=P_{(i-1, j) \rightarrow(i, j)} \pi^{j}(i-1, j)$. Recursively, we can then write that $\pi^{j}(i, j)=P_{(0, j) \rightarrow(i-1, j)} \pi^{j}(0, j)=$ $\prod_{l=1}^{i-1} P(l-1, j) \rightarrow(l, j) \pi^{j}(0, j)$

\section{A.2 Computing the number of cache misses}

It is a fair approximation to assume that, at, the begimning of each subsection, no element is in cache. Therefore. we: write that

$$
\pi^{j}(0, j)=\left(\begin{array}{l}
0 \\
1
\end{array}\right), \forall j \in\left[1, n_{.}\right]
$$

therefore, $\pi_{\text {out }}^{j}(i, j)=P_{(0, j)-(i-1, j)}(2,2)$, and it is now possible to compute $N_{c m}\left(P^{\prime \prime}\right)$ :

$$
N_{c m}\left(P^{\prime \prime}\right)=\sum_{i=1}^{n_{l}} \sum_{j=1}^{n_{c}} \pi_{o u t}^{j}(i, j) \times \nu(i, j)
$$

It is now possible to give an approximation of the total number of cache misses yielded by $A$. The number of cache misses due to a section $S^{\prime}$ of $A^{\prime}$ is given by $N_{c m}\left(S^{\prime}\right)=$ $N_{c m}\left(P_{1}^{\prime \prime}\right)+N_{c m}\left(P_{2}^{\prime \prime}\right)$. Since there are $\frac{N}{C_{s}} N_{c m}\left(S^{\prime}\right)=N^{\prime} \times$ $N_{c m}\left(S^{\prime}\right)$. Now, since there are $C_{S}$ such submatrices in $A$. the total number of cache misses is cqual to $N: \cdots\left(H^{\prime}\right)=$ $C_{S} N_{c m}\left(P^{\prime}\right)$ 
$N_{c m}(P) \simeq \frac{N}{L_{s}}\left(n_{c}^{1} \times n_{l}^{1}+n_{c}^{2} \times n_{l}^{2}\right) p-\frac{1}{2} \frac{N}{l_{s}} \times\left(n_{c}^{1} \times n_{l}^{1} \times\left(n_{l}^{1}-1\right)+n_{c}^{2} \times n_{l}^{2} \times\left(n_{l}^{2}-1\right)\right) \times p^{2}$

Figure 27: Expression of the number of self-interfercuce misses.

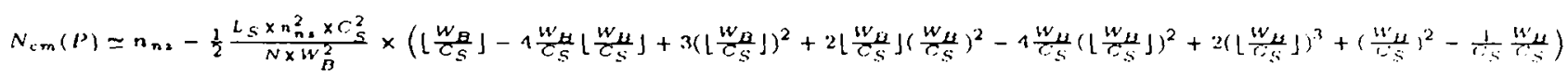

Figure 28: Expression of the number of self-inlerference misses as a function of the problem paramelers.

\section{A.3 Distributions of probability}

U' to now we have considered that each element $(i, j)$ of $A$ had a specific probability $p(i, j)$ of being a non-zero element. This distribution can be non-uniform, i.e $p$ depends effecl.ively on $(i, j)$, or uniform, i.e $p(i, j)=p$. The non-uniform distribution allows a better description of the number of cache misses, but prevents, in most cases, the computation of an analytical expression of this number. The uniform distribution, though less precise, allows to obtain such an axpression.

Non uniform distribution Certain types of sparse marlices cannot be considered to have a uniform distribution within the band. However, in many applications, specifically finite element methods it is a fair approximation to consider that the distribution of probability is constant across the diagonals, that is $p(i, j)=p(i-1, j-1)$. 'This is due to a good repartition of the non-zero elements among the: rows. Indeed, most of the time, the number of non-zero elements per row is constant. And, what is more the distribution of the non-zero elements is relatively similar on all the rows.

leet us now give the expression of the total number of cache misses when the distribution is non-uniform:

$$
\begin{aligned}
& N_{r m}(P)=
\end{aligned}
$$

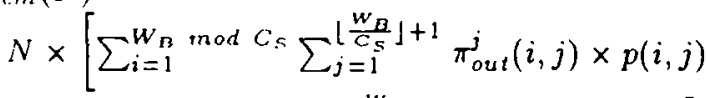

$$
\begin{aligned}
& \left.+\sum_{i=1}^{W_{B}-W_{B} \bmod C_{s}} \sum_{j=1}^{\left\lfloor\frac{\left.W_{B}\right\rfloor}{\left.C_{S}\right\rfloor}\right.} \pi_{\text {out }}^{j}(i, j) \times p(i, j)\right]
\end{aligned}
$$

Therefore, when the distribution is not uniform, the two sums

$$
\sum_{i=1}^{\bmod } C_{S} \sum_{j=1}^{\left\lfloor\frac{W_{B}}{C_{S}}\right\rfloor+1} \pi_{\text {out }}^{j}(i, j) \times p(j)
$$

and

$$
\sum_{i=1}^{W_{B}-W_{B}} \sum_{j=1}^{\bmod C_{S}\left\lfloor\frac{w_{B}}{C_{S}}\right\rfloor} \pi_{\text {out }}^{j}(i, j) \times p(j)
$$

Inust still be computed numerically (this computation is not too costly considering the boundaries of the sums).

Uniform distribution For some sparse applications, it is a reasonnable approximation to consider that the distribution of the non-zero elements is uniform. In that case an analytical expression of the number of cache misses can be derived from the previous formula. Let us show how.

First of all, we compute the uniform probability $p$ that an clement be non-zero. There are $n_{n z}$ non-zero elements in the matrix. Since the number of non-yero elements on each row is constant, we can consider that there is an average: number of $\frac{n_{m}<}{N}$ elements per row. Therefore each element. has a probability $p=\frac{\frac{n_{n}}{y}}{W_{B}}$ of being referenced. Wlien 1,5 is taken into account, the probability that a group of $l . s$ elements of $X$ be referenced is $p=1-\left(1-\frac{\frac{n_{14}}{W_{1 / s}}}{W^{1-s}}\right.$

Let us now give the expression of $P_{(i-1, j)} \rightarrow(i, j)$ :

$$
\begin{array}{ll}
p(l, k) & =p \\
\Rightarrow P_{\text {flush }}(l, k) & =\left(\begin{array}{cc}
1-p & 0 \\
p & 1
\end{array}\right)
\end{array}
$$

and,

$$
P_{\text {load }}(l, k)=\left(\begin{array}{cc}
1 & p \\
0 & 1-p
\end{array}\right)
$$

Then,

$$
\begin{aligned}
& P_{(i-1, j) \rightarrow(i, j)}=\left(P_{\text {flush }}\right)^{n_{c}-1} \times P_{\text {load }} \\
& \Rightarrow P_{(0, j) \rightarrow(i, j)}=\left(\left(P_{\text {flush }}\right)^{n_{c}-1} \times P_{\text {lolat }}\right)^{\prime}
\end{aligned}
$$

And,

$$
\pi^{j}(i, j)=\left(\left(P_{f l u s h}\right)^{n_{c}-1} \times P_{l o u d}\right)^{i} \pi^{j}(0, j)
$$

The explicit expression of $P_{(0, j)-(i, j)}$ is the following

$$
\left(\begin{array}{cl}
\frac{1}{1-(1-p)^{n_{c}}} & {\left[1-(1-p)^{n_{c}-1}\right.} \\
& \left.+p(1-p)^{n_{c}-1}(1-p)^{n_{c}(i-1)}\right]
\end{array}\right)
$$

We can note that $\pi^{j}(i, j)$ is now independent of $j$. What is more, we will consider, without a great loss of precision that:

$$
\pi^{j}(0, j)=\left(\begin{array}{l}
0 \\
1
\end{array}\right)
$$

Therefore, using notations of section 3.1.2, we can dexluce that the total number of cache misses is given by expression of figure A or figure 10.

Now, since $\frac{n_{B}}{N \times W_{B}} \ll 1$, we can write that.

$p \simeq L_{S} \times \frac{n_{n S}}{N \times W_{B}}-L_{S}\left(L_{S}-1\right) \times \frac{n_{n z}^{2}}{N^{2} \times W_{i s}^{2}}+o\left(\frac{n_{n i}}{N \times W_{t S}}\right)$

Therefore, a first order development of expression of $N_{c m}(P)$ (as a function of the problem paramelers) is given by expression of figure $A$.

For sake of simplicity, we can assume thill. $\left\lfloor\frac{W_{u}}{C^{\prime}}\right\rfloor \simeq \frac{H_{u}}{C^{\prime}}$. and therefore:

$$
N_{c m}(P) \simeq n_{n z}-\frac{n_{n z}^{2} \times C_{S}}{2 \times N \times L_{S} \times W_{B}}
$$




\section{A.4 Extending the model to set-associative caches}

The problem formulation for set-associative caches is very similar to the one of direct-mapped caches except that, in the simplified problem, the cache size is now equal to $D_{A} \times L_{S}\left(D_{A}\right.$ is the degree of associativity) instead of $L_{S}$. Therefore, the methodology used for direct-mapped caches can be employed for set-associative caches with some adjustments. The complexity of these modifications depends on the type of replacement policy used inside the cache sets. Considering actual architechures, two replacement policies can be considered: Random Replacement policy or Least Recently Used policy.

Random Replacement For this replacement policy, the main difference lays in the probability of transition matrices. $P_{\text {load }}$ is unchanged but $P_{\text {flush }}$ must be modified. The main difference lays in the fact that a reference is not sufficient for flushing the cache. Several such references should occur before the cache is flushed. This notion can be simply formalized by modifying the probability that. one element be flushed, the expression of which appears in $P_{f l u s h}(1,1)$ and $P_{\text {flush }}(1,3)$.

Least Recently Used In this case, the method previously used changes slightly. More precisely, it is necessary to modify the state vector. Instead of two states only (in,out), we have to consider $D_{A}+1$ states where $D_{A}$ is the degree of associativity. Then, new probability of transition matrices must be computed to describe the probability for an element to shift from one position to another in the LRU cquese. This methodology is the direct extension of the one used for direct-mapped cache where $D_{A}=1$. 


\section{LISTE DES DERNIERES PUBLICATIONS INTERNES IRISA}

PI 642 ARCHE : UN LANGAGE PARALLELE A OBJETS FORTEMENT TYPES Marc BENVENISTE, Valérie ISSARNY

Mars 1992, 132 pages.

PI 643 CARTESIAN AND SATISTICAL APPROACHES OF THE SATISFIABILITY PROBLEM

Israël--César LERMAN

Mars 1992, 58 pages.

PI 644 PRIME MEMORY SYSTEMS DO NOT REQUIRE EUCLIDEAN DIVISION BY A PRIME NUMBER

André SEZNEC, Yvon JEGOU, Jacques LENFANT

Mars 1992, 10 pages.

PI 645 SKEWED-ASSOCIATIVE CACHES

André SEZNEC, François BODIN

Mars 1992, 20 pages.

PI 646 INTERLEAVED PARALLEL SCHEMES : IMPROVING MEMORY THROUGHPUT ON SUPERCOMPUTERS

André SEZNEC, Jacques LENFANT

Mars 1992, 14 pages.

PI 647 COMMUNICATING PROCESSES AND FAULT TOLERANCE : A SHARED MEMORY MULTIPROCESSOR EXPERIENCE

Michel BANATRE, Maurice JEGADO, Philippe JOUBERT, Christine MORIN

Mars 1992, 40 pages.

PI 648 SET-THEORETIC GRAPH REWRITING

Jean-Claude RAOULT, Frédéric VOISIN

Mars 1992, 18 pages.

PI 649 UNE STRUCTURE D'INFORMATION POUR LES ALGORITHMES

D'EXCLUSION MUTUELLE FONDES SUR UNE ARBORESCENCE

Jean-Michel HELARY, Achour MOSTEFAOUI, Michel RAYNAL

Mars 1992, 18 pages.

PI 650 BLOCK-ARNOLDI AND DAVIDSON METHODS FOR UNSYMMETRIC LARGE EIGENVALUE PROBLEMS

Miloud SADKANE

Avril 1992, 24 pages.

PI 651 COMPILING SEQUENTIAL PROGRAMS FOR DISTRIBUTED MEMORY

PARALLEL COMPUTERS WITH PANDORE II

Françoise ANDRE, Olivier CHERON, Jean-Louis PAZAT

Avril 1992, 18 pages.

PI 652 CHARACTERIZING THE BEHAVIOR OF SPARSE ALGORITHMS ON CACHES Olivier TEMAM, William JALBY

Avril 1992, 20 pages.

PI 653 MADMACS : UN OUTIL DE PLACEMENT ET ROUTAGE POUR LE DESSIN DE MASQUES DE RESEAUX REGULIERS

Eric GAUTRIN, Laurent PERRAUDEAU, Oumarou SIE

Avril 1992,16 pages. 
ISSN 0249-6399 